\title{
Generation of correlation and covariance matrices for the recommended temporal DN parameters in 6- and 8-group models
}

\author{
Vladimir M. Piksaikin, Andrey S. Egorov, Dmitrii E. Gremyachkin*, Konstantin V. Mitrofanov, \\ and Vjacheslav F. Mitrofanov \\ JSC "SSC RF - IPPE", Bondarenko sq. 1, Obninsk, 249033, Russian Federation
}

Received: 20 December 2019 / Received in final form: 11 March 2020 / Accepted: 18 June 2020

\begin{abstract}
In the present work the new data on correlation and covariance matrices have been calculated for all recommended data on temporal parameters of delayed neutrons in 6- and 8-group models. The method for producing the correlation and covariance data for the temporal DN parameters is described. The table of changes is listed in the text. Some examples of the data generated on basis of experimental DN decay curves (for IPPE data) and data generated on the basis of decay curves restored using temporal parameters (for works of another authors) are listed too.
\end{abstract}

\section{Introduction}

Following Tuttle's evaluation of delayed neutron (DN) data in 1979 [1], OECD/NEA WPEC Subgroup (SG) 6 performed an evaluation with the aim of improving the macroscopic DN data for the major actinide nuclides ${ }^{235} \mathrm{U},{ }^{238} \mathrm{U}$ and ${ }^{239} \mathrm{Pu}$, which are of primary interest for reactor technology [2]. Recommended data were provided for the total DN yields for the above three nuclides, and for the temporal DN parameters in the 6and 8-group models for the whole set of nuclides [3] included in Tuttle's evaluation [1]. However, SG 6 had no intention to elaborate an approach for calculating the correlation and the covariance data for DN temporal parameters.

In 2013 the Nuclear Data Section of the IAEA initiated a Coordinated Research Project (CRP) with the purpose to create a Reference Database for both microscopic and macroscopic DN databased on the latest experimental efforts [4]. Among the numerous new DN data developed in the frame of this project, are the revised recommended temporal DN parameters for both the 6- and 8-group models. The revised DN group-parameters database contained information on correlations and covariances only for a few nuclides, and mainly for the 6-group model which were obtained on the basis of the IPPE data. Taking into account up-to-date requirements from the reactor community, there is a need to extend such

\footnotetext{
* e-mail: dgremyachkin@ippe.ru
}

information for all the nuclides included in the new recommended DN database produced under the CRP project.

The purpose of this work is threefold: (1) to develop an approach for calculation of the correlation and the covariance data for the temporal DN parameters both in the 6- and the 8-group models; (2) to calculate the correlation and the covariance matrices for all nuclides included in the recommended IAEA/CRP database [5]; (3) to provide this data in a suitable form for easy inclusion in the online IAEA/NDS beta-delayed neutron database.

\section{Methods for estimation of DN temporal parameters and generation of the correlation and the covariance data in the 6 - and 8-group models}

\subsection{The IPPE method for estimation of DN parameters and their correlation and covariance data in the 6- and 8-group models}

The DN temporal parameters (relative abundances and half-lives) are determined on the basis of the DN decay curves obtained in measurements of DN activity as a function of the time after irradiation of sample by neutrons. The expectation of the number of counts $z_{i}$ detected in the time interval $\left(t_{i-1}, t_{i}\right)$ in the cyclic measurements with the irradiation time $t_{i r r}, N$ cycles each of which has duration $T$ and the neutron background intensity $B$ can be represented 
by the following expression [6]

$$
\begin{aligned}
M\left(z_{i}\right)= & A \cdot \sum_{k=1}^{K} p_{k} \cdot \tau_{k} \cdot\left(1-e^{-\frac{t_{i r r}}{\tau_{k}}}\right) \cdot\left(1-e^{-\frac{t_{i}-t_{i-1}}{\tau_{k}}}\right) \cdot e^{-\frac{t_{i-1}-t_{i r r}}{\tau_{k}}} \\
& \cdot\left[\frac{N}{1-e^{-\frac{T}{\tau_{k}}}}-\frac{e^{-\frac{T}{\tau_{k}}}}{\left(1-e^{-\frac{T}{\tau_{k}}}\right)^{2}} \cdot\left(1-e^{-\frac{N \cdot T}{\tau_{k}}}\right)\right] \\
& +B \cdot\left(t_{i}-t_{i-1}\right) \cdot N
\end{aligned}
$$

where $A$ - the DN saturation activity, $K-$ the number of DN groups, $p_{k}-$ the probability that precursors belong to the $k$ th DN group, $\tau_{k}-$ mean life-time of precursors corresponding to $k$ th DN group $\left(\tau_{k}=1 / \lambda_{k}=T_{k} / \ln 2, \lambda_{k}\right.$ and $T_{k}$ being the decay constant and half-life of the $k$ th DN group, respectively). The numbers $z_{i}$ can be considered as independent random variables with the mathematical expectation that depends on $n=2 \cdot K+2$ unknown parameters

$$
\left\{A, B, p_{k}, \tau_{k}, k=1, \ldots, K\right\}
$$

From the physical point of view, all parameters in set (2) must be non-negative. The $p_{k}$ parameters should be normalized to unity. Therefore, it is suitable to make the following parameterization

$$
p_{k}=\frac{y_{k}}{\sum_{j=1}^{k} y_{j}} \text {. }
$$

If one determines the value of one of the group $y_{k}$ as equal to unity (usually this is the longest lived group of delayed neutron precursors) then the remaining $y_{k}$ will represent the relative yields of DN with respect to this group. Then set (2) is transformed to the following set

$$
\left\{A, B, y_{k}, \tau_{k}, k=1, \ldots, K\right\}
$$

all elements of which are non-negative and one of the $y_{k}$ is equal to unity. It is convenient to introduce for set (4) a new designation $a_{j}$ by renumbering them from 1 to $n$ and considering them as the coordinates of vector $\vec{a}$. Having the explicit expression

$$
M\left(z_{i}\right)=F_{i}\left(a_{1}, \ldots, a_{n}\right)
$$

one may formulate the task of the estimation of parameters $\left\{a_{1}, \ldots, a_{n}\right\}$ as the search for an optimal description of the measured values $z_{i}$ by the functions $F_{i}(\vec{a})$. Statistically the iteration process can be formulated in the following manner. The hypothesis $H_{0}$ is made that the values of the parameters are

$$
\left\{a_{j}=a_{j}^{(0)}, j=1, \ldots, n\right\}
$$

An alternative hypothesis $H$ is that the parameters are presented in the following form

$$
\left\{a_{j}=a_{j}{ }^{(0)} \cdot e^{\delta_{j}}, j=1, \ldots, n\right\} .
$$

By expanding $F_{i}(\vec{a})$ in a Taylor's series in the vicinity of $\vec{\delta}=\{\delta j=0, j=1, \ldots, n\}$ the linear model with respect to the corrections $\vec{\delta}$ is created

$$
F_{i}(\vec{a})=F_{i}\left(\vec{a}^{(0)}\right)+\sum_{j=1}^{n} c_{i j} \cdot \delta_{j}
$$

where

$$
c_{i j}=\left.\frac{\partial F_{i}(\vec{a})}{\partial a_{j}} \cdot \frac{\partial a_{j}}{\partial \delta_{j}}\right|_{\delta_{j}=0} .
$$

This model is used for the estimation of $\vec{\delta}$ on the basis of measured values $z_{i}$. If the obtained corrections considerably differ from zero then the hypothesis $H_{0}$ is discarded and a corrected hypothesis $H$ is proposed. If the difference of the corrections from zero is considered as negligible then the hypothesis $H_{0}$ is accepted and the vector $\vec{a}^{(0)}$ is chosen as the resultant estimation. The statistical uncertainties of the estimation are determined by the uncertainty of the correction term. The present approach in solving the problem is that the estimation of the vector $\vec{\delta}$ is determined by means of the sequence of estimations $\left\{\vec{\delta}^{(i)}, i=1, \ldots, m\right\}$, each of which uses only one measurement $z_{i}$ and the previous estimation.

Particularly for each $i$ one should find $\vec{\delta}^{(i)}$ from the condition of the minimum of the following quadratic form

$$
\begin{aligned}
Q\left(\vec{\delta}^{(i)}\right)= & \left(\vec{\delta}^{(i)}-\vec{\delta}^{(i-1)}\right)^{T} \cdot V_{i-1}^{-1} \cdot\left(\vec{\delta}^{(i)}-\vec{\delta}^{(i-1)}\right) \\
& +w_{i} \cdot\left[z_{i}-F_{i}\left(\vec{a}^{(0)}\right)-c_{i}^{T} \cdot \vec{\delta}^{(i)}\right]^{2}
\end{aligned}
$$

where $V_{i-1}^{-1}-$ matrix that is the inverse to the covariance matrix $V_{i-1}$ of the estimation $\vec{\delta}^{(i-1)}, c_{i}^{T}-i$ th row of matrix $C$. From the Gauss-Markov theorem about the estimation of the generalized least squares method corresponding to the minimum of the $Q\left(\vec{\delta}^{(i)}\right)$ value, one can obtain the following formulas for estimation of the value of delayed neutron parameters as well as appropriate covariance matrix

$$
\begin{gathered}
\vec{\delta}^{(i)}=\vec{\delta}^{(i-1)}+\frac{z_{i}-F_{i}\left(\vec{a}^{(0)}\right)-c_{i}^{T} \cdot \vec{\delta}^{(i)}}{D\left(z_{i}\right)+c_{i}^{T} \cdot V_{i-1} \cdot c_{i}} \cdot V_{i-1} \cdot c_{i}, \\
V_{i}=V_{i-1}-\frac{\left(V_{i-1} \cdot c_{i}\right) \cdot\left(V_{i-1} \cdot c_{i}\right)^{T}}{D\left(z_{i}\right)+c_{i}^{T} \cdot V_{i-1} \cdot c_{i}}
\end{gathered}
$$

where $D\left(z_{i}\right)$-the variance of $z_{i}$.

The correlation matrix was obtained with the following formula

$$
\operatorname{Cor}\left(x_{i}, x_{j}\right)=\frac{\operatorname{Cov}\left(x_{i}, x_{j}\right)}{\sigma\left(x_{i}\right) \cdot \sigma\left(x_{j}\right)},
$$


where $i, j=1, \ldots, n ; \sigma\left(x_{i}\right), \sigma\left(x_{j}\right)$ - variances of parameters $x_{i}$ and $x_{j}$, respectively.

There are two types of DN data sets in the recommended IAEA/CRP database. The first one is related to the DN parameter sets for which experimental decay curves are available (the IPPE data). These data can be used for generating covariance data directly from the least square estimation procedure applied to the decay curves. The second type of data comprise the DN parameter sets without the associated experimental decay curves. In each case we used a different approach for generation of the correlation and the covariance data.

\subsection{The LANL method for estimation of DN parameters in 8-group model}

Instead of using original DN decay curve or its analytical estimation incorporated in the IPPE expansion method, the LANL techniques is based on another analytical function - the simulation of the time-dependence of relative power of a system containing a nuclide under investigation. A detailed description of this method is presented in paper [3]. For reader convenience main features of this method are described below.

Firstly a series of 20 transients of systems corresponding to step inputs of reactivity in a range from $\$ 0.01$ to $\$ 0.95$ is generated on the basis of the exact solution of the point-reactor kinetic equations according the function

$$
N=\sum_{j=1}^{n+1} A_{j} \cdot \exp \left(\omega_{j} \cdot t\right)
$$

where $N$ - the relative neutron power; $A_{j}$ - the amplitude of the $j$ th term; $\omega_{j}-$ the $j$ th root of the inhour equation calculated using the original DN parameters; $n$ - the number of DN groups in the original model. The amplitudes, $A_{j}$, in this equation are related to the relative abundance, $a_{i}$, and decay constant of each group, $\lambda_{i}$, and to the system reactivity, $\rho_{\$}$, as follows

$$
A_{j}=\frac{\rho_{\$}}{\left[\frac{\Lambda}{\beta}+\sum_{i=1}^{n} \frac{a_{i} \cdot \lambda_{i}}{\left(\omega_{j}+\lambda_{i}\right)^{2}}\right] \cdot \omega_{j}},
$$

where $\Lambda$ - the neutron generation time; $\beta$ - the effective delayed neutron fraction.

Based on the obtained dependences of the relative neutron flux on the time $N(t)$ and the asymptotic inverse period, a file of initial data was created for a specific set of group parameters of the DN, which were then used to obtain the group parameters of the DN in the framework of the 8-group model. In this case, the relative neutron flux was described by a function similar to equation (14), in which the amplitudes were determined by expression

$$
A_{j}=\frac{\omega_{a} \cdot \frac{\Lambda}{\beta}+\sum_{i=1}^{m} \frac{a_{i} \cdot \omega_{a}}{\left(\omega_{a}+\lambda_{i}\right)}}{\left[\frac{\Lambda}{\beta}+\sum_{i=1}^{m} \frac{a_{i} \cdot \lambda_{i}}{\left(\omega_{j}+\lambda_{i}\right)^{2}}\right] \cdot \omega_{j}},
$$

Table 1. The recommended data set of the 8-group DN parameters for fission of ${ }^{240} \mathrm{Pu}$ by fast neutrons expanded from the 6-group model (the LANL expansion method [3]).

The LANL expansion method. Pu-240 fast: Fast

Spectrum, 8-groups DN parameters, Keepin et al. (1957) [7].

\begin{tabular}{lllll}
\hline Gr. No., $i$ & $\begin{array}{l}T(i), \\
\text { Half-lives, } s\end{array}$ & $\begin{array}{l}a(i), \\
\text { Rel. Abun. }\end{array}$ & \pm & 1 sigma \\
\hline 1 & 55.6 & 0.022 & \pm & 0.003 \\
2 & 24.5 & 0.207 & \pm & 0.005 \\
3 & 16.3 & 0.080 & \pm & 0.002 \\
4 & 5.21 & 0.161 & \pm & 0.055 \\
5 & 2.37 & 0.314 & \pm & 0.009 \\
6 & 1.04 & 0.105 & \pm & 0.010 \\
7 & 0.424 & 0.079 & \pm & 0.017 \\
8 & 0.195 & 0.032 & \pm & 0.003 \\
\hline
\end{tabular}

Average Half-life: $\operatorname{Sum}\left[a(i)^{*} T(i)\right]=9.32 \pm 0.66 \mathrm{~s}$.

where $\omega_{a}$ - the asymptotic inverse period corresponding to the reactivity value obtained on the basis of the initial model of DN characteristics.

In the process of this procedure, the time dependence of the characteristics of the hypothetical system, the scale of the reactivity change (only positive values are considered) and the average half-life of the DN remain unchanged. It should be noted that there is no uncertainties associated with the decay constant in the 8-group model (the values of the half-lives are fixed). Therefore all of the uncertainties in the reactivity are lumped into the uncertainties of group abundances.

\subsection{Comparison of the IPPE and the LANL methods for estimation of the 8-group DN characteristics}

The discussed expansion processes were compared using the experimental 6-group data obtained by Keepin et al. [7] from the fast neutron induced fission of ${ }^{240} \mathrm{Pu}$. The results of the LANL expansion method was taken from the recommended DN data sets presented in paper [3] and shown in Table 1. The results of the estimation of the 8group DN parameters obtained by the IPPE method are presented in Table 2.

It is seen from Tables 1 and 2 that the 8 -group relative abundances obtained by the LSF processing of the model decay curves based on the 6-group DN data by both methods agree within their uncertaities. This is a good indication that the correlation matrix obtained in the procedure of estimating the 8-group DN parameters is correct. Taking into account that the LANL method includes the procedure for the estimation of uncertainties which is consistent with the uncertainties of the original 6 group DN data sets the covariance matrix for the 8-group model in case of DN datasets without experimental decay curves was calculated on the basis of the IPPE correlaton matrix and the uncertainties provided in the recommended 8-group datasets by Spriggs et al. [3] (see appropriate paragraph below). 
Table 2. The 8-group DN parameters from processing of the DN decay curve generated from the 6-group DN parameters from fission of ${ }^{240} \mathrm{Pu}$ by fast neutrons (the IPPE expansion method [6]).

Results of estimation 8-group DN parameters by the IPPE method: $\mathrm{Pu}-240$ fast: Fast Spectrum. Keepin et al. (1957) [7].

\begin{tabular}{lllll}
\hline Gr. No., $i$ & $\begin{array}{l}T(i), \\
\text { Half-lives, } s\end{array}$ & $\begin{array}{l}a(i), \\
\text { Rel. Abun. }\end{array}$ & \pm & 1 sigma \\
\hline 1 & 55.6 & 0.0231 & \pm & 0.0004 \\
2 & 24.5 & 0.196 & \pm & 0.003 \\
3 & 16.3 & 0.093 & \pm & 0.002 \\
4 & 5.21 & 0.149 & \pm & 0.003 \\
5 & 2.37 & 0.321 & \pm & 0.004 \\
6 & 1.04 & 0.105 & \pm & 0.003 \\
7 & 0.424 & 0.080 & \pm & 0.004 \\
8 & 0.195 & 0.033 & \pm & 0.002 \\
\hline
\end{tabular}

Average half-life: $\operatorname{Sum}\left[a(i)^{*} T(i)\right]=9.29 \pm 0.16 \mathrm{~s}$.

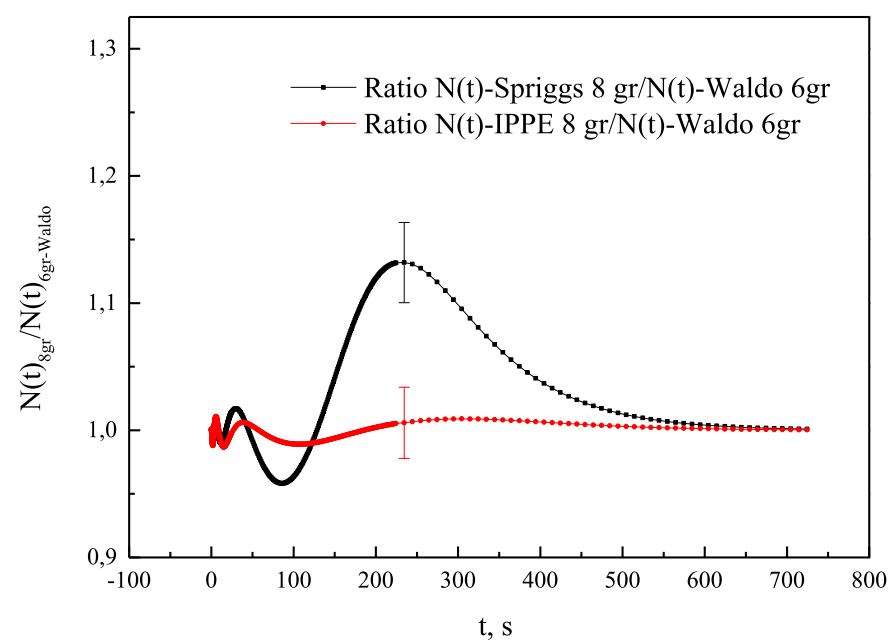

Fig. 1. The ratio of the decay curves calculated on the basis of the 8-group DN dataset with the LANL and the IPPE expansion methods to the DN decay curve obtained with the original 6-group data for ${ }^{242} \mathrm{Pu}$ by Waldo [8].

It should be noted that a disagreement in the expanded 8-group DN parameters (listed in [3]) and the original 6-group DN data sets was found for the fast neutron induced fission of ${ }^{242} \mathrm{Pu}[8]$ and ${ }^{238} \mathrm{U}[7]$. Namely, there is no agreement between the 8-group data obtained by the LANL expansion method [3] and the data obtained with the help of the IPPE method [6]. This means that the estimated correlation matrix will be not suitable for the recommended 8-group DN dataset. In order to investigate the reason for such discrepancy the ratios of the decay curves obtained for the 8-group DN datasets by the LANL and the IPPE expansion methods to the DN decay curves obtained with the original 6-group data by Waldo $\left({ }^{242} \mathrm{Pu}\right.$. [8]) were calculated. The resulting data are plotted in Figure 1.

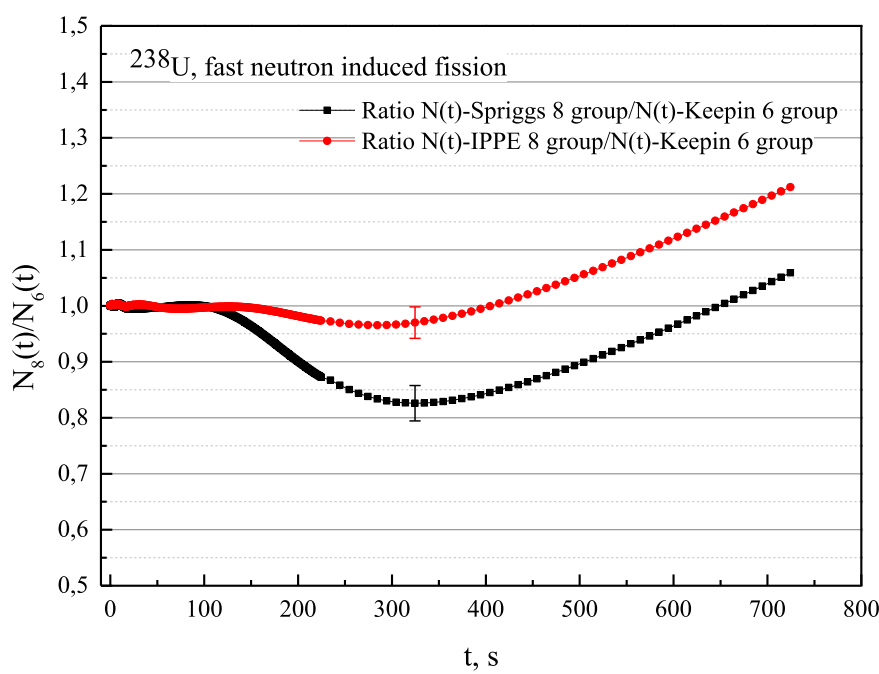

Fig. 2. The ratio of the decay curves calculated on the basis of the 8-group DN dataset with the LANL and the IPPE expansion methods to the DN decay curve obtained with the original 6-group data for ${ }^{238} \mathrm{U}$ by Keepin [7].

In the same way the ratios of the decay curves were obtained for the 8-group DN data set using the LANL and the IPPE expansion methods to the DN decay curve from the fast neutron fission of ${ }^{238} \mathrm{U}$ obtained with the original 6group data by Keepin [7] were calculated. Results are shown in Figure 2.

It is seen from Figure 1 that the decay curve calculated with the Spriggs' 8-group data set signicantly differs from the original 6 -group decay curves by Waldo [8]. The decay curve obtained with the IPPE methods does not differ from the Waldo's original decay curve by more than $5 \%$. As a result, it was decided to replace the 8-group DN dataset for the fast neutron-induced fission of ${ }^{242} \mathrm{Pu}$ [8] obtained with the help of the LANL expansion method by the corresponding DN dataset estimated with the IPPE method.

From Figure 2 one can see that the Spriggs recommended 8-group data set [3] in the range from 160 to $580 \mathrm{~s}$ significantly differ from the original 6-group data by Keepin [7]. At the same time the decay curve obtained using the IPPE method is in the well consistency with the original Keepin's data in the range below 500 s. Inconsistency in the range above $500 \mathrm{~s}$ could be due to the discrepancy between the half-lives of the first (longest) DN group for the 6- and 8-group representation $(53.0 \mathrm{~s}$ and $55.6 \mathrm{~s}$, respectively). This issue is scrutinized in the work devoted to the results of IAEA/CRP [5].

\section{Generation of correlation and covariance data}

\subsection{Generation of correlation and covariance data for the IPPE data}

The correlation and covariance matrices were generated for the following the IPPE DN datasets obtained in the thermal, fast and high energy neutron induced fission which is considered as new sets of recommended data [5]: 
[9];

${ }^{232} \mathrm{Th}$ high: $E_{n}=14.23 \mathrm{MeV}$, Roshchenko et al. (2010)

${ }^{233} \mathrm{U}$ thermal: Thermal Spectrum, Piksaikin et al. (2002) [10];

${ }^{233} \mathrm{U}_{\text {_fast: }} E_{n}=0.59 \mathrm{MeV}$, Piksaikin et al. (1997) [11];

${ }^{235} \mathrm{U}^{-}$thermal: Thermal spectrum, Piksaikin et al. (2002) $[\overline{6}]$

${ }^{235} \mathrm{U}$ fast: $E_{n}=0.81 \mathrm{MeV}$, Piksaikin et al. (2002) [6];

${ }^{236} \mathrm{U}$ - fast: $E_{n}=3.7 \mathrm{MeV}$, Piksaikin et al. (1997) [13];

${ }^{238} \mathrm{U}^{-}$fast: $E_{n}=3.75 \mathrm{MeV}$, Piksaikin et al. (2002) [6];

[14];

${ }^{38} \mathrm{U}^{-}$high: $E_{n}=14.23 \mathrm{MeV}$, Piksaikin et al. (2007) [12];

${ }^{37} \mathrm{~Np}_{-}$fast: $E_{n}=3.745 \mathrm{MeV}$, Piksaikin et al. (1997)

${ }^{237} \mathrm{~Np}$ high: $E_{n}=14.23 \mathrm{MeV}$, Gremyachkin et al., (2017) $[1 \overline{5]}$;

${ }^{239} \mathrm{Pu}$ thermal: Thermal spectrum, Piksaikin et al.

(2013) [1] ;

${ }^{239} \mathrm{Pu}$ fast: $E_{n}=0.86 \mathrm{MeV}$, Piksaikin et al. (2013) [11];

${ }^{239} \mathrm{Pu}$ - high: $E_{n}=15.8 \mathrm{MeV}$, Piksaikin et al.(2007) [16];

${ }^{241} \mathrm{Am}$ - fast: $E_{n}=1.06 \mathrm{MeV}$, Piksaikin et al.(2013) [11];

${ }^{241} \mathrm{Am}$ high: $E_{n}=15.8 \mathrm{MeV}$, Gremyachkin et al. (2018) [17].

\subsubsection{The 6-group model of DN parameters}

The 6-group model was used in the primary processing of the IPPE experimental data with the above discussed method (see Sect. 2.1). The decay curves obtained in the experiments with the irradiation times of 180 or $300 \mathrm{~s}$ were used for the estimation of the DN parameters for the four longest groups. The parameters of the remaining two groups were estimated from the decay curves corresponding to experiments with the irradiation time of $15 \mathrm{~s}$.

The averaging procedure was used when several datasets of DN parameters were available for specified incident neutron energy. The procedure for averaging several number of DN parameter sets is described in the paper [6]. All runs in our experiments can be considered as independent. There is no parameter which is derived from one run and then used in processing of the other one. Background $\mathrm{B}$, activity $\mathrm{A}$ and corresponding uncertainties $d A$ and $d B$ are estimated for each decay curve simultaneously with $a_{i}$ and $T_{i}$ and their uncertainties $d a_{i}$ and $d T_{i}$. If we have the vector $\vec{b}^{(\imath)}$, components of which are the DN parameters

$\vec{b}^{(i)} \equiv\left(b_{j}{ }^{(i)}, j=1, \ldots, 2 \cdot K\right) \equiv\left(Y_{1}^{(i)}, \ldots, Y_{K}{ }^{(i)}, T_{1}{ }^{(i)}, \ldots, T_{K}^{(i)}\right)$.

In a $K$-group model approximation and the appropriate covariance matrix $V_{j}$ for each experimental run $j$, one can obtain the resulting set $\vec{b}$ of the relative abundances and periods averaged over $M$ independent measurements according to the following expression

$$
\vec{b}=V\left(V_{1}^{-1} \cdot \vec{b}^{(1)}+V_{2}^{-1} \cdot \vec{b}^{(2)}+\cdots+V_{M}^{-1} \cdot \vec{b}^{(M)}\right),
$$

where $V=\left(V_{1}^{-1}+V_{2}^{-1}+\ldots+V_{M}^{-1}\right)^{-1}$ is the covariance matrix of the set $\vec{b}$ of the DN parameters. The uncertainties
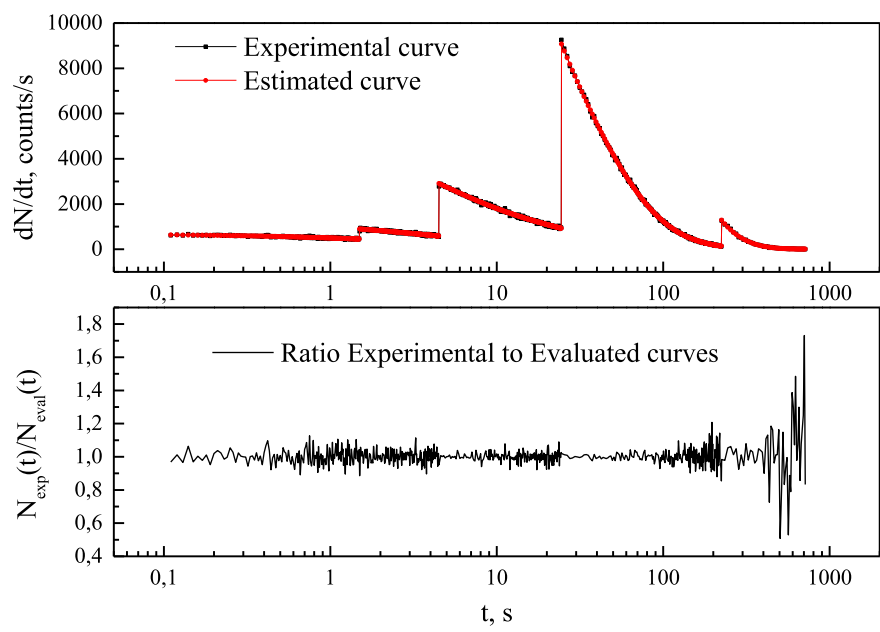

Fig. 3. Time dependence of the delayed neutron intensity. Squares - experimental curve, circles - curve estimated in the process of least square fit. Lower graph shows the ratio of the experimental to evaluated curve.

of the averaged values of the relative abundances and the half-lives of DN were taken as the square root of appropriate diagonal elements of the covariance matrix $V$.

Thus, the DN temporal parameters and appropriate correlation and covariance matrices in the 6-group model for the IPPE data were obtained directly from experimental decay curves using the averaging procedure which takes into account the strong cross-correlation between the DN parameters derived from the least squares fitting of the decay curves.

\subsubsection{The 8-group model of DN parameters}

There are two possible approaches for generation of the DN parameters $\left(a_{i}, T_{i}\right)$, correlation and covariance data for the 8-group model. The first one is to estimate these data directly from the LSF processing of experimental decay curves. The second one is to produce these data from the LSF processing of the decay curves calculated using the 6group DN parameters $\left(a_{i}, T_{i}\right)$ estimated in the primary processing of experimental data (a model decay curve).

The outlined approaches were compared using experimental decay curve obtained in the experiment in which ${ }^{235} \mathrm{U}$ sample was irradiated by thermal neutrons during $180 \mathrm{~s}$. The DN counting time interval was $0.1-720 \mathrm{~s}$. The total number of counts registered by the neutron detector was 840272 . In Figure 3 this experimental decay curve is shown together with decay curve calculated with the help of the estimated 6-group DN parameters $\left(a_{i}, T_{i}\right)$. The both DN delayed curves were the LSF processed with purpose to estimate the 8-group DN parameters $\left(a_{i}\right)$ and appropriate correlation matrices. The two sets of the DN parameters obtained in the LSF processing of the experimental data and the estimated decay curve are listed in Tables 3 and 4, respectively. The correlation matrices for the sets of DN parameters are listed in Tables 5 and 6.

The comparison of the sets of the parameters, $a_{i}$, and their uncertainties, $d a_{i}$, as well as the values of the 
Table 3. The 8-group DN parameters $\left(a_{i}\right)$ obtained in processing of the experimental decay curve measured from fission of ${ }^{235} \mathrm{U}$ by thermal neutrons.

\begin{tabular}{|c|c|c|c|c|}
\hline \multirow[t]{2}{*}{ No. of DN group, $j$} & \multicolumn{2}{|c|}{$\begin{array}{l}\text { Activity A, counts/s }-5550.54 \pm 24.06 \\
\text { Background B, counts/s }-0.0176 \pm 0.0017\end{array}$} & \multirow{2}{*}{$\begin{array}{l}\text { Rel. abundance } \\
a_{i} \\
\end{array}$} & \multirow{2}{*}{$\begin{array}{l}\text { Rel. abundance } \\
\text { uncertainty } \\
d a_{i} \\
\end{array}$} \\
\hline & Half-life $T, \mathrm{~s}$ & $d T, \mathrm{~s}$ & & \\
\hline 1 & 55.6 & 0 & 0.03346 & 0.00066 \\
\hline 2 & 24.5 & 0 & 0.14607 & 0.00287 \\
\hline 3 & 16.3 & 0 & 0.10357 & 0.00191 \\
\hline 4 & 5.21 & 0 & 0.19028 & 0.00391 \\
\hline 5 & 2.37 & 0 & 0.32783 & 0.00397 \\
\hline 6 & 1.04 & 0 & 0.09057 & 0.00339 \\
\hline 7 & 0.424 & 0 & 0.08127 & 0.00378 \\
\hline \multirow[t]{2}{*}{8} & 0.195 & 0 & 0.02696 & 0.00134 \\
\hline & Average half-life, s & $9.029 \pm 0.088$ & & \\
\hline
\end{tabular}

Table 4. The 8-group DN parameters $\left(a_{i}\right)$ obtained in the LSF processing of the decay curve calculated with the help of the estimated 6-group DN parameters.

\begin{tabular}{|c|c|c|c|c|}
\hline \multirow[t]{2}{*}{ No. of DN group, $j$} & \multicolumn{2}{|c|}{$\begin{array}{l}\text { Activity A, counts/s }-5548.75 \pm 24.29 \\
\text { Background B, counts/s }-0.0176 \pm 0.0017^{*}\end{array}$} & \multirow{2}{*}{$\begin{array}{l}\text { Rel. abundance } \\
a_{i}\end{array}$} & \multirow{2}{*}{$\begin{array}{l}\text { Rel. abundance } \\
\text { uncertainty } \\
d a_{i} \\
\end{array}$} \\
\hline & Half-life $T, \mathrm{~s}$ & $d T, \mathrm{~s}$ & & \\
\hline 1 & 55.6 & 0 & 0.03331 & 0.00064 \\
\hline 2 & 24.5 & 0 & 0.14782 & 0.00286 \\
\hline 3 & 16.3 & 0 & 0.10076 & 0.00192 \\
\hline 4 & 5.21 & 0 & 0.19518 & 0.0039 \\
\hline 5 & 2.37 & 0 & 0.32043 & 0.00394 \\
\hline 6 & 1.04 & 0 & 0.09465 & 0.0035 \\
\hline 7 & 0.424 & 0 & 0.08452 & 0.00392 \\
\hline \multirow[t]{2}{*}{8} & 0.195 & 0 & 0.02332 & 0.00116 \\
\hline & Average Half-life, s & $9.031 \pm 0.088$ & & \\
\hline
\end{tabular}

* - Parameters A (activity) and B (background) and their uncertainties have been estimated in the LSF fit together with all the other DN parameters. All these data included in the correlation matrix (see Appendix A for detailed discussion on the a1 and da1. Full matrix is presented there). However we did not included all correlation coefficients in the final matrix because these data are related to specific experiments and is not really needed for implementation of DN parameters. But they are certainly obtained with taking into account parameters A and B.

correlation coefficients, $\operatorname{Cor}\left(a_{i}, a_{j}\right)$, obtained in the both approaches shows very good agreement of the data. The most important feature of the obtained results is the identity of the correlation matrices and the structure of the parameter uncertainties in the both approaches. It means that the statistical dispersion of the decay curves has no main influence on the correlation properties of DN parameters at least in the case of good statistical accuracy of experimental decay curve and the reliable estimation of the DN parameter values. Based on these results we have used the second approach for the estimation of the DN temporal data and their correlation and covariance matrices for the IPPE data. Although the possibility to obtain these data on the basis of the IPPE experimental data is still remain.
Thus, the 8-group DN parameters (the relative abundances, the correlation and the covariance matrices) were obtained in the following approach. Firstly, the DN decay curves were calculated using the evaluated 6-group data sets $\left(\left\langle a_{i}\right\rangle,\left\langle T_{i}\right\rangle\right)$ obtained from the primary processing of the experimental data including averaging procedure. Then these decay curves were analyzed using the iterative least-squares method to obtain the relative abundances, the correlation and the covariance matrices in the 8-group model representation (see Appendix B for the motivations of using such a complicated approach).

The revisions of the DN parameters sets made on the basis of the experimental IPPE data in the frame of the IAEA/CRP (Nuclear Data Sheets, to be published) with respect to the recommended sets of the temporary DN parameters presented in [3] are shown in Table 7. 
Table 5. The 8-group correlation matrix obtained in processing of the experimental decay curve measured from fission of ${ }^{235} \mathrm{U}$ by thermal neutrons.

\begin{tabular}{|c|c|c|c|c|c|c|c|c|c|c|c|c|c|c|c|c|}
\hline & a1 & $\mathrm{T} 1$ & $\mathrm{a} 2$ & $\mathrm{~T} 2$ & a3 & $\mathrm{T} 3$ & $\mathrm{a} 4$ & $\mathrm{~T} 4$ & a5 & $\mathrm{T} 5$ & a6 & $\mathrm{T} 6$ & a7 & $\mathrm{T} 7$ & a8 & $\mathrm{T} 8$ \\
\hline a1 & 1 & & & & & & & & & & & & & & & \\
\hline $\mathrm{T} 1$ & 0 & 1 & & & & & & & & & & & & & & \\
\hline $\mathrm{a} 2$ & 0 & 0 & 1 & & & & & & & & & & & & & \\
\hline $\mathrm{T} 2$ & 0 & 0 & 0 & 1 & & & & & & & & & & & & \\
\hline a3 & 0 & 0 & -0.70 & 0 & 1 & & & & & & & & & & & \\
\hline T3 & 0 & 0 & 0 & 0 & 0 & 1 & & & & & & & & & & \\
\hline a4 & 0 & 0 & 0.54 & 0 & -0.7 & 0 & 1 & & & & & & & & & \\
\hline $\mathrm{T} 4$ & 0 & 0 & 0 & 0 & 0 & 0 & 0 & 1 & & & & & & & & \\
\hline a5 & 0 & 0 & 0.22 & 0 & 0.19 & 0 & -0.57 & 0 & 1 & & & & & & & \\
\hline T5 & 0 & 0 & 0 & 0 & 0 & 0 & 0 & 0 & 0 & 1 & & & & & & \\
\hline $\mathrm{a} 6$ & 0 & 0 & 0.05 & 0 & -0.04 & 0 & 0.18 & 0 & -0.42 & 0 & 1 & & & & & \\
\hline T6 & 0 & 0 & 0 & 0 & 0 & 0 & 0 & 0 & 0 & 0 & 0 & 1 & & & & \\
\hline a7 & 0 & 0 & 0.04 & 0 & -0.03 & 0 & 0.1 & 0 & -0.14 & 0 & -0.12 & 0 & 1 & & & \\
\hline $\mathrm{T} 7$ & 0 & 0 & 0 & 0 & 0 & 0 & 0 & 0 & 0 & 0 & 0 & 0 & 0 & 1 & & \\
\hline a8 & 0 & 0 & 0.01 & 0 & -0.01 & 0 & 0.01 & 0 & -0.01 & 0 & -0.02 & 0 & -0.02 & 0 & 1 & \\
\hline T8 & 0 & 0 & 0 & 0 & 0 & 0 & 0 & 0 & 0 & 0 & 0 & 0 & 0 & 0 & 0 & 1 \\
\hline
\end{tabular}

Table 6. The 8-group correlation matrix obtained in the LSF processing of the decay curve calculated with the help of the estimated 6-group DN parameters (a model decay curve).

\begin{tabular}{|c|c|c|c|c|c|c|c|c|c|c|c|c|c|c|c|c|}
\hline & $\mathrm{a} 1$ & $\mathrm{~T} 1$ & $\mathrm{a} 2$ & $\mathrm{~T} 2$ & a3 & $\mathrm{T} 3$ & $\mathrm{a} 4$ & $\mathrm{~T} 4$ & a5 & $\mathrm{T} 5$ & $\mathrm{a} 6$ & $\mathrm{~T} 6$ & a7 & $\mathrm{T} 7$ & a8 & $\mathrm{T} 8$ \\
\hline a1 & 1 & & & & & & & & & & & & & & & \\
\hline $\mathrm{T} 1$ & 0 & 1 & & & & & & & & & & & & & & \\
\hline $\mathrm{a} 2$ & 0 & 0 & 1 & & & & & & & & & & & & & \\
\hline $\mathrm{T} 2$ & 0 & 0 & 0 & 1 & & & & & & & & & & & & \\
\hline a3 & 0 & 0 & -0.69 & 0 & 1 & & & & & & & & & & & \\
\hline T3 & 0 & 0 & 0 & 0 & 0 & 1 & & & & & & & & & & \\
\hline $\mathrm{a} 4$ & 0 & 0 & 0.54 & 0 & -0.7 & 0 & 1 & & & & & & & & & \\
\hline $\mathrm{T} 4$ & 0 & 0 & 0 & 0 & 0 & 0 & 0 & 1 & & & & & & & & \\
\hline a5 & 0 & 0 & 0.22 & 0 & 0.19 & 0 & -0.56 & 0 & 1 & & & & & & & \\
\hline $\mathrm{T} 5$ & 0 & 0 & 0 & 0 & 0 & 0 & 0 & 0 & 0 & 1 & & & & & & \\
\hline $\mathrm{a} 6$ & 0 & 0 & 0.05 & 0 & -0.04 & 0 & 0.18 & 0 & -0.42 & 0 & 1 & & & & & \\
\hline $\mathrm{T} 6$ & 0 & 0 & 0 & 0 & 0 & 0 & 0 & 0 & 0 & 0 & 0 & 1 & & & & \\
\hline a7 & 0 & 0 & 0.04 & 0 & -0.03 & 0 & 0.11 & 0 & -0.14 & 0 & -0.12 & 0 & 1 & & & \\
\hline $\mathrm{T} 7$ & 0 & 0 & 0 & 0 & 0 & 0 & 0 & 0 & 0 & 0 & 0 & 0 & 0 & 1 & & \\
\hline a8 & 0 & 0 & 0.01 & 0 & 0 & 0 & 0.01 & 0 & -0.01 & 0 & -0.02 & 0 & -0.02 & 0 & 1 & \\
\hline $\mathrm{T} 8$ & 0 & 0 & 0 & 0 & 0 & 0 & 0 & 0 & 0 & 0 & 0 & 0 & 0 & 0 & 0 & 1 \\
\hline
\end{tabular}

\subsection{Generation of correlation and covariance data for DN datasets without experimental DN decay curves}

The correlation and the covariance matrices both in the 6and 8-group model were generated for the following original DN data sets presented as the recommended data [3] and which included in new recommended DN data sets without any changes [5]:

${ }^{232}$ Th fast: Fast Spectrum, 6-groups, Keepin et al. (1957) [7];
${ }^{231} \mathrm{~Pa}$ fast: Above Cd Cutoff, 6-groups, Anoussis et al. (1973) [18̄;

${ }^{231} \mathrm{~Pa}$ high: $E_{n}=14.8 \mathrm{MeV} \max$, 4-groups, Brown et al. (1971) [19];

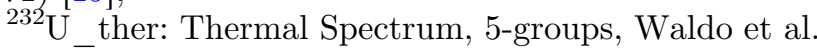
(1981) $[\overline{8}]$;

[20];

${ }^{233}{ }_{\text {U_high: }} E_{n}=14.7 \mathrm{MeV}$, 6-groups, East et al. (1970) [20];

${ }^{235} \mathrm{U}_{-}$high: $E_{n}=14.7 \mathrm{MeV}, 6$-groups, East et al. (1970) 
Table 7. The revisions of the DN parameters sets made in the frame of the IAEA/CRP project made on the basis of the experimental IPPE data.

\begin{tabular}{|c|c|c|c|}
\hline \multirow[t]{2}{*}{ Target nuclide } & \multicolumn{3}{|c|}{ Neutron energy } \\
\hline & Thermal & Fast & High \\
\hline${ }^{232} \mathrm{Th}$ & - & No revision & New (6 and 8-group data) \\
\hline${ }^{233} \mathrm{U}$ & New (6 and 8-group data) & New (6 and 8-group data) & No revision \\
\hline${ }^{235} \mathrm{U}$ & New (6 and 8-group data) & New (6 and 8-group data) & No revision \\
\hline${ }^{236} \mathrm{U}$ & - & New (6 and 8-group data) & - \\
\hline${ }^{238} \mathrm{U}$ & - & New (6 and 8-group data) & New (6 and 8-group data) \\
\hline${ }^{237} \mathrm{~Np}$ & - & New (6 and 8-group data) & New (6 and 8-group data) \\
\hline${ }^{239} \mathrm{Pu}$ & New (6 and 8-group data) & New (6 and 8-group data) & New (6 and 8-group data) \\
\hline${ }^{241} \mathrm{Am}$ & No revision & New (6 and 8-group data) & New (6 and 8-group data) \\
\hline${ }^{242} \mathrm{Pu}$ & & New (8-group) & No revision \\
\hline
\end{tabular}

${ }^{238} \mathrm{Pu}$ ther: Thermal Spectrum, 6-groups, Waldo et al. (1981) [8];

${ }^{238} \mathrm{Pu}$ fast: Fast Spectrum, 5-groups, Benedetti et al. (1982) $[2 \overline{1}]$;

${ }^{240} \mathrm{Pu}$ fast: Fast Spectrum, 6-groups, Keepin et al. (1957) $[7 \overline{]}$ [22];

${ }^{241} \mathrm{Pu}$ ther: Thermal Spectrum, 5-groups, Cox (1961)

${ }^{241} \mathrm{Pu}$ fast: $\sim$ Fission Spectrum, 6-groups, Gudkov et al. (19899) [23];

${ }^{242} \mathrm{Pu}$ fast: Fast Spectrum, 6-groups, Waldo et al. (1981) [8];

${ }^{242} \mathrm{Pu}$ high: $E n=14.7 \mathrm{MeV}$, 6-groups, East et al. (1970) $[2 \overline{0]}$;

${ }^{24} \mathrm{Am}$ ther: Thermal Spectrum, 6-groups, Waldo et al. (198̄) [8];

${ }^{242 \mathrm{~m}} \mathrm{Am}$ ther: Thermal Spectrum, 6-groups, Waldo et al. (1981) [8];

${ }^{243} \mathrm{Am}$ fast: Fast Spectrum, 7-groups, Charlton et al. (1998) [24];

${ }^{245} \mathrm{Cm}$ ther: Thermal Spectrum, 6-groups, Waldo et al. (1981) [8];

${ }^{249} \mathrm{Cf}$ ther: Thermal Spectrum, 4-groups, Waldo et al. (1981) [8];

${ }^{252} \mathrm{Cf}$ spon: Spontaneous Fission, 4-groups, Chulick et al. (1969) [25].

As a first step, the DN decay curves were generated on the basis of the available the 6-group DN parameters (some of these data are available in the 4- and 5-group models $\left[{ }^{231} \mathrm{~Pa}\right.$ etc.]). The following additional parameters were used to generate the decay curves: the irradiation time $180 \mathrm{~s}$; the number of cycles - 1 ; the time sequence of delayed neutron counting $-0.01 \mathrm{~s}-150$ channels, $0.02 \mathrm{~s}-$ 150 channels, $0.1 \mathrm{~s}-200$ channels, $1 \mathrm{~s}-200$ channels, $10 \mathrm{~s}$ - 50 channels; the activity A - 10000 counts/s; the neutron background $\mathrm{B}-10$ counts/s (see Appendix $\mathrm{C}$ for conditions $\mathrm{A}=1000,5000 ; \mathrm{B}=100)$. The obtained $\mathrm{DN}$ decay curves were used to estimate the DN correlation and covariance data for both the 6- and 8-group model.

To justify this approach for generation of correlation matrices for the 6-group data we have made the following estimations. As a first step, we have obtained the delayed neutron parameters $\left(a_{i}, T_{i}\right)$ and correlation matrix using the experimental decay curve for the thermal neutron induced fission of ${ }^{235} \mathrm{U}$ (see the above discussion on the estimation of the 8-group DN parameters for the IPPE data). On the second step we have estimated delayed neutron parameters and correlation matrice on the basis of the model decay curve generated with $\left(a_{i}, T_{i}\right)$ obtained on the first step of processing. The obtained data on the temporal DN parameters $\left(a_{i}, T_{i}\right)$ are presented in Tables 8 and 9 for the experimental and the model decay curves, respectively.

The obtained correlation matrices for the 6-group representation are shown in Tables 10 and 11.

It is seen from Tables 8 and 9 that the relative abundances, $a_{i}$, and their uncertainties, $d a_{i}$, obtained in processing of the experimental decay curve and the model decay curve calculated with estimated DN parametes are in excellent agreement. The difference in the correlation coefficients $\left(\operatorname{Cor}\left(a_{i}, a_{j}\right), \operatorname{Cor}\left(a_{i}, T_{j}\right), \operatorname{Cor}\left(T_{i}, T_{j}\right)\right)$ in appropriate correlation matrices is negligible. Thus in the 6group model as well as in the case of the 8-group model (see discussion above) one can see the identity of the DN characteristics obtained in the LSF processing of experimental and corresponding model decay curves. Moreover one can see the identical correlation properties of DN parameters in the experimental and model decay curves. This is an important condition for obtaining a reliable correlation matrix which corresponds to the values of the DN neutron parameters that are close to the original DN data. In order to save uncertainties of DN parametes cited in original papers the covariance data were obtained using the correlation matrix derived in the LSF estimation of the $D N$ parameters and the uncertainties $\sigma\left(a_{i}\right)$ and $\sigma\left(T_{i}\right)$ taken from the original group data sets (quoted in the published papers):

$$
\begin{gathered}
\operatorname{Cov}\left(a_{i}, T_{j}\right)=\operatorname{Cor}\left(a_{i}, T_{j}\right) \cdot \sigma\left(a_{i}\right) \cdot \sigma\left(T_{j}\right), \\
\operatorname{Cov}\left(a_{i}, a_{j}\right)=\operatorname{Cor}\left(a_{i}, a_{j}\right) \cdot \sigma\left(a_{i}\right) \cdot \sigma\left(a_{j}\right), \\
\operatorname{Cov}\left(T_{i}, T_{j}\right)=\operatorname{Cor}\left(T_{i}, T_{j}\right) \cdot \sigma\left(T_{i}\right) \cdot \sigma\left(T_{j}\right) .
\end{gathered}
$$


Table 8. The 6-group DN parameters $\left(a_{i}, T_{i}\right)$ obtained in processing of the experimental decay curve measured from fission of ${ }^{235} \mathrm{U}$ by thermal neutrons.

\begin{tabular}{|c|c|c|c|c|}
\hline \multirow[t]{2}{*}{ No. of group, $j$} & \multicolumn{2}{|c|}{$\begin{array}{l}\text { Activity A, counts } / \mathrm{s}-5548.16 \pm 31.85 \\
\text { Background B, counts/s }-0.232 \pm 0.007\end{array}$} & \multirow{2}{*}{$\begin{array}{l}\text { Rel. abundance } \\
a_{i}\end{array}$} & \multirow{2}{*}{$\begin{array}{l}\text { Rel. abundance uncertainty } \\
d a_{i}\end{array}$} \\
\hline & Half-life $T, \mathrm{~s}$ & $d T, \mathrm{~s}$ & & \\
\hline 1 & 55.66249 & 0.35406 & 0.0340 & 0.0008 \\
\hline 2 & 22.41893 & 0.17516 & 0.2205 & 0.0049 \\
\hline 3 & 6.27864 & 0.19257 & 0.1956 & 0.0083 \\
\hline 4 & 2.2793 & 0.0483 & 0.3894 & 0.0140 \\
\hline 5 & 0.60662 & 0.02928 & 0.1173 & 0.0055 \\
\hline \multirow[t]{2}{*}{6} & 0.22951 & 0.01144 & 0.0432 & 0.0022 \\
\hline & Average Half-life, s & $9.033 \pm 0.349$ & & \\
\hline
\end{tabular}

Table 9. The 6-group DN parameters $\left(a_{i}, T_{i}\right)$ obtained in the LSF processing of the model decay curve calculated with the help of the 6-group DN parameters estimated on the basis of experimental decay curve.

\begin{tabular}{|c|c|c|c|c|}
\hline \multirow[t]{2}{*}{ No. of group, $j$} & \multicolumn{2}{|c|}{$\begin{array}{l}\text { Activity A, counts/s }-5547.86 \pm 31.86 \\
\text { Background B, counts/s }-0.24 \pm 0.01 \\
\end{array}$} & \multirow{2}{*}{$\begin{array}{l}\text { Rel. abundance } \\
a_{i} \\
\end{array}$} & \multirow{2}{*}{$\begin{array}{l}\text { Rel. abundance uncertainty } \\
d a_{i}\end{array}$} \\
\hline & Half-life $T, \mathrm{~s}$ & $d T$ & & \\
\hline 1 & 55.84012 & 0.35724 & 0.03361 & 0.00074 \\
\hline 2 & 22.49088 & 0.17577 & 0.21999 & 0.00485 \\
\hline 3 & 6.31886 & 0.19381 & 0.19645 & 0.00831 \\
\hline 4 & 2.27236 & 0.04815 & 0.39028 & 0.01404 \\
\hline 5 & 0.60668 & 0.02928 & 0.11687 & 0.00552 \\
\hline \multirow[t]{2}{*}{6} & 0.22994 & 0.01146 & 0.04279 & 0.00213 \\
\hline & Average Half-life, s & $9.034 \pm 0.350$ & & \\
\hline
\end{tabular}

Table 10. The 6-group correlation matrix obtained in processing of the experimental decay curve measured from fission of ${ }^{235} \mathrm{U}$ by thermal neutrons.

\begin{tabular}{|c|c|c|c|c|c|c|c|c|c|c|c|c|c|c|}
\hline & A & B & a1 & $\mathrm{T} 1$ & $\mathrm{a} 2$ & $\mathrm{~T} 2$ & a3 & T3 & $\mathrm{a} 4$ & $\mathrm{~T} 4$ & a 5 & $\mathrm{~T} 5$ & a6 & $\mathrm{T} 6$ \\
\hline $\bar{A}$ & 1 & & & & & & & & & & & & & \\
\hline B & 0.02 & 1 & & & & & & & & & & & & \\
\hline a1 & 0 & 0 & 1 & & & & & & & & & & & \\
\hline $\mathrm{T} 1$ & -0.31 & -0.07 & 0 & 1 & & & & & & & & & & \\
\hline $\mathrm{a} 2$ & -0.31 & -0.05 & 0 & 0.86 & 1 & & & & & & & & & \\
\hline $\mathrm{T} 2$ & -0.24 & -0.01 & 0 & 0.51 & 0.31 & 1 & & & & & & & & \\
\hline a3 & -0.04 & -0.02 & 0 & 0.38 & 0.4 & 0.34 & 1 & & & & & & & \\
\hline $\mathrm{T} 3$ & -0.12 & 0 & 0 & 0.23 & -0.07 & 0.68 & -0.3 & 1 & & & & & & \\
\hline $\mathrm{a} 4$ & -0.16 & -0.03 & 0 & 0.68 & 0.57 & 0.7 & -0.04 & 0.68 & 1 & & & & & \\
\hline $\mathrm{T} 4$ & -0.35 & 0 & 0 & 0.06 & 0.06 & 0.04 & -0.63 & 0.25 & 0.17 & 1 & & & & \\
\hline a5 & 0.33 & -0.01 & 0 & 0.14 & 0.14 & 0.11 & -0.02 & 0.06 & -0.04 & 0.32 & 1 & & & \\
\hline T5 & -0.42 & 0 & 0 & 0.04 & 0.03 & 0.04 & 0 & 0.04 & -0.12 & 0.14 & 0.03 & 1 & & \\
\hline a6 & 0.25 & 0 & 0 & 0.03 & 0.03 & 0.02 & 0 & 0.01 & 0.01 & 0.05 & -0.03 & 0.02 & 1 & \\
\hline $\mathrm{T} 6$ & -0.12 & 0 & 0 & 0.02 & 0.03 & 0.02 & 0 & 0.01 & 0 & 0.06 & -0.02 & 0.01 & -0.01 & 1 \\
\hline
\end{tabular}


Table 11. The 6-group correlation matrix obtained in the LSF processing of the model decay curve calculated with the help of the estimated 6-group DN parameters from fission of ${ }^{235} \mathrm{U}$ by thermal neutrons.

\begin{tabular}{llllllllllllll}
\hline & $\mathrm{A}$ & $\mathrm{B}$ & $\mathrm{a} 1$ & $\mathrm{~T} 1$ & $\mathrm{a} 2$ & $\mathrm{~T} 2$ & $\mathrm{a} 3$ & $\mathrm{~T} 3$ & $\mathrm{a} 4$ & $\mathrm{~T} 4$ & $\mathrm{a} 5$ & $\mathrm{~T} 5$ & $\mathrm{a} 6$ \\
\hline $\mathrm{A}$ & 1 & & & & & & & & & & & & $\mathrm{~T} 6$ \\
$\mathrm{~B}$ & 0.02 & 1 & & & & & & & & & & & \\
$\mathrm{a} 1$ & 0 & 0 & 1 & & & & & & & & & & \\
$\mathrm{~T} 1$ & -0.31 & -0.07 & 0 & 1 & & & & & & & & \\
$\mathrm{a} 2$ & -0.31 & -0.05 & 0 & 0.86 & 1 & & & & & & & & \\
$\mathrm{~T} 2$ & -0.24 & -0.01 & 0 & 0.51 & 0.31 & 1 & & & & & & & \\
$\mathrm{a} 3$ & -0.04 & -0.02 & 0 & 0.38 & 0.4 & 0.34 & 1 & & & & & & \\
$\mathrm{~T} 3$ & -0.12 & 0 & 0 & 0.23 & -0.07 & 0.68 & -0.3 & 1 & & & & & \\
$\mathrm{a} 4$ & -0.16 & -0.03 & 0 & 0.68 & 0.57 & 0.7 & -0.04 & 0.68 & 1 & & & & \\
$\mathrm{~T} 4$ & -0.35 & 0 & 0 & 0.06 & 0.06 & 0.04 & -0.63 & 0.25 & 0.17 & 1 & & & \\
$\mathrm{a} 5$ & 0.33 & -0.01 & 0 & 0.14 & 0.14 & 0.11 & -0.02 & 0.06 & -0.04 & 0.32 & 1 & & \\
$\mathrm{~T} 5$ & -0.42 & 0 & 0 & 0.04 & 0.03 & 0.04 & 0 & 0.04 & -0.12 & 0.14 & 0.03 & 1 & \\
$\mathrm{a} 6$ & 0.25 & 0 & 0 & 0.03 & 0.03 & 0.02 & 0 & 0.01 & 0.01 & 0.05 & -0.03 & 0.02 & 1 \\
$\mathrm{~T} 6$ & -0.12 & 0 & 0 & 0.02 & 0.03 & 0.02 & 0 & 0.01 & 0 & 0.06 & -0.02 & 0.01 & -0.01 \\
\hline
\end{tabular}

Table 12. Relative abundances and half-life of delayed neutrons (6-group DN data set) for fast neutron induced fission of ${ }^{241} \mathrm{Pu}$.

Pu-241 fast: Fission Spectrum. 6-groups. Gudkov et al. (1989) [23]

\begin{tabular}{lllllll}
\hline \multicolumn{7}{c}{ OriginalModel:6groups } \\
\hline Gr. No. $i$ & $T(i)$. Half-lives. s & \pm & 1 sigma & $a(i)$. Rel. Abun. & \pm & 1 sigma \\
\hline 1 & 55.9 & \pm & 0.000 & 0.017 & \pm & 0.003 \\
2 & 23.0 & \pm & 0.000 & 0.218 & \pm & 0.022 \\
3 & 5.64 & \pm & 0.000 & 0.179 & \pm & 0.019 \\
4 & 2.10 & \pm & 0.000 & 0.362 & \pm & 0.040 \\
5 & 0.578 & \pm & 0.000 & 0.189 & \pm & 0.042 \\
6 & 0.201 & \pm & 0.000 & 0.035 & \pm & 0.014 \\
\hline
\end{tabular}

Ave.rage Half-life: $\operatorname{Sum}\left[\mathrm{a}(i)^{*} \mathrm{~T}(i)\right]=7.85 \pm .54 \mathrm{~s}$.

The 8-group data on temporal DN parameters and the appropriate correlation matrices have been obtained in the same way as it have been shown for the 6-group data. The covariance matrices have been produced using the uncertainties $\sigma\left(a_{i}\right)$ from the data recommended by Spriggs [3].

\section{The IAEA reference database for beta-delayed neutron emission}

Numerical information on the corelation and the covariance data for the temporal DN parameters in the 6- and 8-group model as well as new recommended values of the relative abundances and the half-lives are presented in the IAEA Reference Database for Beta-Delayed Neutron
Emission [26]). These data should be considered as a partial re-evaluation and extension of the recommended data sets presented in Spriggs et al. paper [3].

The IAEA DN data base includes the following information for the nuclides which were investigated experimentally:

- Relative abundances and half-life of delayed neutrons (6-group DN data sets);

- Correlation matrix (6-group DN data sets);

- Covariance matrix (6-group DN data sets);

- Relative abundances and half-life of delayed neutrons (8-group DN data sets);

- Correlation matrix (8-group DN data sets);

- Covariance matrix (8-group DN data sets).

As an example all the DN data sets for fission of ${ }^{241} \mathrm{Pu}$ by fast neutrons [23] are shown in Tables 12-17. 
Table 13. Relative abundances and half-life of delayed neutrons (8-group DN data set) for fast neutron induced fission of ${ }^{241} \mathrm{Pu}$.

Expanded model using a fixed set of half-lives: 8 groups

Pu-241_fast: Fission Spectrum. Gudkov et al. (1989) [23]

\begin{tabular}{lllll}
\hline Gr. No. $i$ & $T(i)$. Half-lives. s & $a(i)$. Rel. Abun. & \pm & 1 sigma \\
\hline 1 & 55.6 & 0.016 & \pm & 0.002 \\
2 & 24.5 & 0.175 & \pm & 0.019 \\
3 & 16.3 & 0.055 & \pm & 0.012 \\
4 & 5.21 & 0.170 & \pm & 0.018 \\
5 & 2.37 & 0.280 & \pm & 0.035 \\
6 & 1.04 & 0.166 & \pm & 0.033 \\
7 & 0.424 & 0.113 & \pm & 0.035 \\
8 & 0.195 & 0.024 & \pm .006 \\
\hline
\end{tabular}

Average Half-life: $\operatorname{Sum}\left[a(i)^{*} T(i)\right]=7.85 \pm .54 \mathrm{~s}$.

Table 14. Correlation matrix for the 6-group DN data set obtained for fast neutron induced fission of ${ }^{241} \mathrm{Pu}$.

\begin{tabular}{|c|c|c|c|c|c|c|c|c|c|c|c|c|}
\hline & a1 & $\mathrm{T} 1$ & $\mathrm{a} 2$ & $\mathrm{~T} 2$ & a3 & T3 & $\mathrm{a} 4$ & $\mathrm{~T} 4$ & a5 & T5 & $\mathrm{a} 6$ & $\mathrm{~T} 6$ \\
\hline a1 & 1 & & & & & & & & & & & \\
\hline $\mathrm{T} 1$ & 0 & 1 & & & & & & & & & & \\
\hline $\mathrm{a} 2$ & 0 & 0 & 1 & & & & & & & & & \\
\hline $\mathrm{T} 2$ & 0 & 0 & 0 & 1 & & & & & & & & \\
\hline a3 & 0 & 0 & 0.12 & 0 & 1 & & & & & & & \\
\hline T3 & 0 & 0 & 0 & 0 & 0 & 1 & & & & & & \\
\hline $\mathrm{a} 4$ & 0 & 0 & 0.68 & 0 & -0.46 & 0 & 1 & & & & & \\
\hline $\mathrm{T} 4$ & 0 & 0 & 0 & 0 & 0 & 0 & 0 & 1 & & & & \\
\hline a5 & 0 & 0 & 0.08 & 0 & 0.36 & 0 & -0.43 & 0 & 1 & & & \\
\hline $\mathrm{T} 5$ & 0 & 0 & 0 & 0 & 0 & 0 & 0 & 0 & 0 & 1 & & \\
\hline a6 & 0 & 0 & 0.01 & 0 & 0.01 & 0 & 0 & 0 & -0.08 & 0 & 1 & \\
\hline T6 & 0 & 0 & 0 & 0 & 0 & 0 & 0 & 0 & 0 & 0 & 0 & 1 \\
\hline
\end{tabular}

\section{Conclusions}

We have presented the new data on the correlation and the covariance matrices for temporal DN parameters (the relative abundances and half-lives) that were generated in the 6- and 8-group model presentation for all nuclides included in the recommended data of the IAEA Reference Database for Beta-Delayed Neutron Emission [26]. Relative abundances and half-life of delayed neutrons (6-group DN data sets);

- Correlation matrix (6-group DN data sets);

- Covariance matrix (6-group DN data sets);

- Relative abundances and half-life of delayed neutrons (8-group DN data sets);

- Correlation matrix (8-group DN data sets);

- Covariance matrix (8-group DN data sets).

There are two types of DN data sets in the recommended IAEA database. The first one is related to the DN parameter sets for which experimental decay curves are available (the IPPE data). The second type of data comprise the DN parameter sets without the associated experimental decay curves. In each case we used a different approach for generation of the correlation and the covariance data.

The DN temporal parameters and appropriate correlation and covariance matrices in the 6-group model for the IPPE data were obtained directly from experimental decay curves using the averaging procedure which takes into account the strong cross-correlation between the DN parameters derived from the least squares fitting of the decay curves.

The 8-group DN parameters (the relative abundances, the correlation and the covariance matrices) for the IPPE data sets were obtained in the following approach. Firstly, the DN decay curves were calculated using the evaluated 6group data sets $\left(\left\langle a_{i}\right\rangle,\left\langle T_{i}\right\rangle\right)$ obtained from the primary processing of the experimental data including averaging procedure. Then these decay curves were analyzed using the iterative least-squares method to obtain the relative abundances, the correlation and the covariance matrices in the 8-group model representation. 

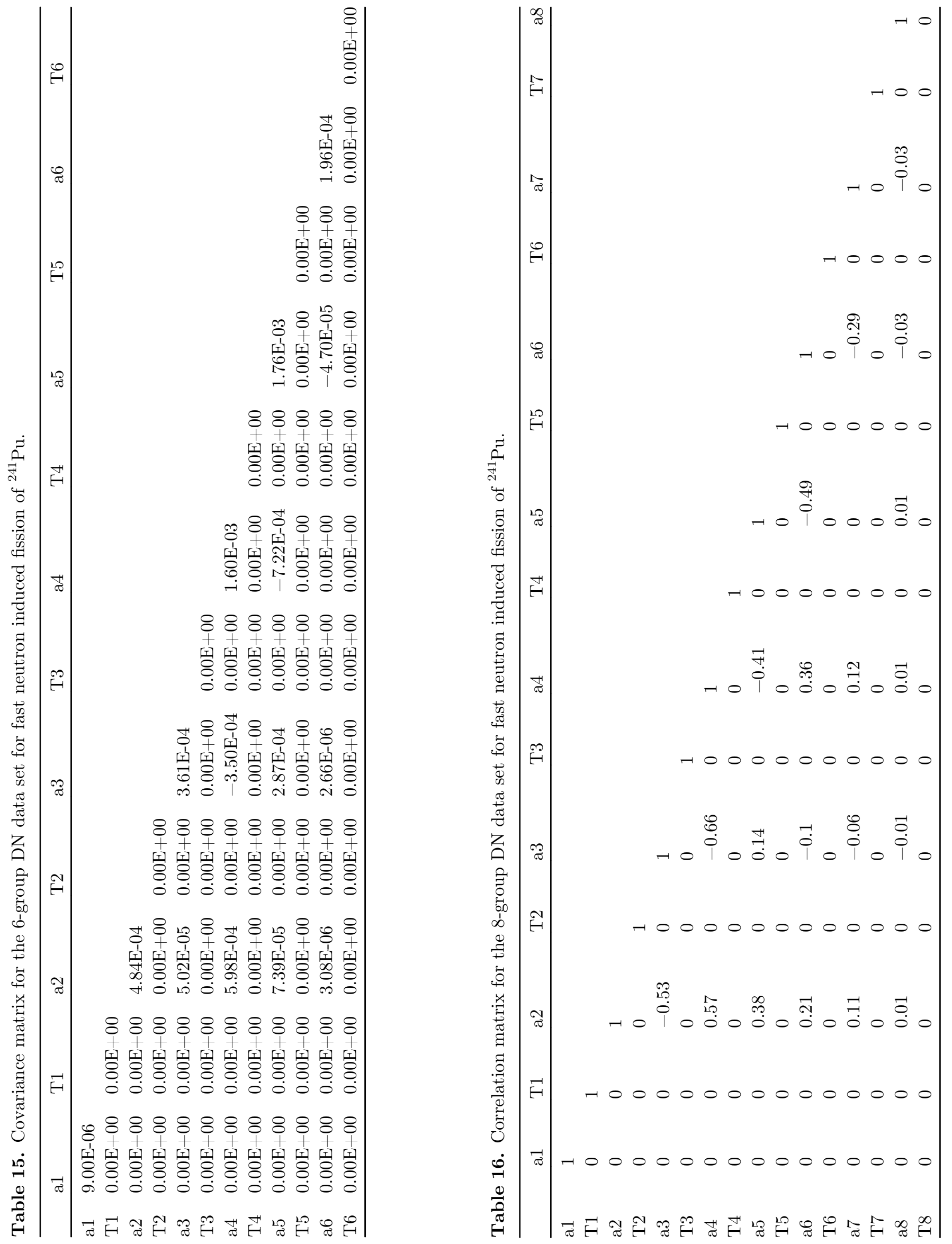
The revisions of the DN parameters sets including generation of the correlation and the covariance data made on the basis of the experimental IPPE data with respect to the recommended sets of the temporary DN parameters presented in [3] are shown in following Table 7.

Generation of the correlation and the covariance data for the DN sets without experimental decay curve was made on the basis of the model decay curves obtained using the values of the original data on relative abandances and half-lives. The values of the relative abundances and the half-lives as well as their uncertainties for these DN data sets remain unchanged.

Justification of such approach was made in a special study. It was shown the identity of the correlation matrices, DN parameters and the structure of the parameter uncertainties obtained in the LSF processing of experimental decay curve and corresponding model decay curve both for the 6 - and 8-group models. It means that the statistical dispersion of the decay curves has no main influence on the correlation properties of DN parameters.

This work was performed under an SSA with the IAEA/Nuclear Data Section on New aggregate data in the IAEA Reference Database for beta-delayed neutron emission (Reference: TALNAPC20190118-001). Authors are appreciated P. Dimitriou from Nuclear Data Section of the IAEA for initialization of the present work and useful discussions during its preparation.

\section{Author contribution statement}

Development of the approach and algorithm for analysis of covariance data for the temporary delayed neutron parameters (V. Piksaikin - 70\%, D. Gremyachkin - 30\%). Development of the computer codes for processing of the experimental and evaluated delayed neutron decay curves (A. Egorov - 60\%, V. Mitrofanov $40 \%$ ). Processing of the experimental and evaluated delayed neutron data with the purpose to obtain covariance and correlation data (K. Mitrofanov - 50\%, D. Gremyachkin $50 \%$ ). Preparation of the manuscript (V. Piksaikin - 60\%, D. Gremyachkin - 40\%).

\section{References}

1. R.J. Tuttle, Review of delayed neutron yields in nuclear fission in Yields in Nuclear Fission. in: Proc. Consultants' Mtg. on Delayed Neutron Properties. Report INDC(NDS)10\%. IAEA. Vienna. Austria, August 1979, p. 29

2. D'Angelo, Overview of the delayed neutron data activities and results monitored by the NEA/WPEC Subgroup 6, Prog. Nucl. Energy 41, 5. G.D. 3 (2002)

3. G.D. Spriggs, J.M. Campbell, V.M. Piksaikin, An 8-group delayed neutron model based on a consistent setof half-lives, Prog. Nucl. Energy 41, 223 (2002)

4. I. Dillmann, P. Dimitriou, B. Singh, Development of ReferenceDatabaseforBeta-delayedneutronemission, IAEA report INDC(NDS)-0643, 2014

5. P. Dimitriou, I. Dillmann, B. Singh, V. Piksaikin, K.P. Rykaczewski, J. Tain, A. Algora, I.N. Borzov, D. Cano-Ott, S. Chiba, A. Egorov, D. Foligno, D. Gremyachkin, T. 
Marketin, F. Minato, K.V. Mitrofanov, CRP members in alphabetical order, et al., Development of a Reference Database for Beta-Delayed Neutron Emission. NUCLEAR DATA SHEETS, to be published

6. V.M. Piksaikin, L.E. Kazakov, S.G. Isaev, M.Z. Tarasko, V.A. Roshchenko, R.G. Tertytchnyi, G.D. Spriggsand J. M. Campbell, Energy dependence of relative abundances and periods of delayed neutrons from neutron-induced fission of U-235, U-238, $\mathrm{Pu}-239$ in 6- and 8-group model representation, Progr. Nucl. Energy 41, 203 (2002)

7. G. Keepin, T. Wimett, R. Zeigler, Delayed neutrons from fissionable isotopes of uranium, plutonium and thorium, Phys. Rev. 107, 1044 (1957)

8. R.W. Waldo. R.A. Karam, R.A. Meyer, Delayed neutron yields: time dependent measurements and a predictive model, Phys. Rev. C 23, 1113 (1981)

9. V.A. Roshchenko, V.M. Piksaikin, et al., Temporary characteristics of delayed neutrons and partial cross sections of emissive fission in fission of ${ }^{232} \mathrm{Th}$ by neutrons in the energy range from 3.2-17.9 MeV, Phys. At. Nucl. 73, 913 (2010)

10. V.M. Piksaikin, L.E. Kazakov, et al., Relative yield and period of individual groups of delayed neutrons in ${ }^{233} \mathrm{U},{ }^{235} \mathrm{U}$ and ${ }^{239} \mathrm{Pu}$ fission by epithermal neutrons, At. Energy 92, 147 (2002)

11. V.M. Piksaikin, A.S. Egorov, K.V. Mitrofanov, The absolute total delayed neutron yields, relative abundances and halflife of delayed neutron groups from neutron induced fission of ${ }^{232} \mathrm{Th},{ }^{233} \mathrm{U},{ }^{236} \mathrm{U},{ }^{239} \mathrm{Pu}$ and ${ }^{241} \mathrm{Am}$, Report INDC(NDS)0646, IAEA, Vienna, Austria, 2013

12. V.M. Piksaikin, Ju.F. Balakshev, S.G. Isaev, L.E. Kazakov, G.G. Korolev, B.D. Kuzminov, N.N. Sergachev, M.Z. Tarasko, Measurements of periods, relative abundances and absolute total yields of delayed neutrons from fast neutron induced fission of ${ }^{235} \mathrm{U}$ and ${ }^{237} \mathrm{~Np}$, in Conf. Proc. Nucl. Data for Science and Technology, Triest, Italy (1997), vol. 59 , p. 485

13. S.G. Isaev, V.M. Piksaikin, L.E. Kazakov, M.Z. Tarasko, Energy dependence of average half-life of delayed neutron precursors in fast neutron induced fission of ${ }^{235} \mathrm{U}$ and ${ }^{236} \mathrm{U}$, in Proc. XIVth Int. Workshop on Nuclear Fission Physics, Obninsk, Russia, 12-15 October 1998, p. 257

14. V.M. Piksaikin, V.A. Roshchenko, G.G. Korolev, Relative yield of delayed neutrons and half-life of their precursor nuclei from ${ }^{238} \mathrm{U}$ fission by $14.2-17.9 \mathrm{MeV}$ neutrons, At. Energy 102, 124 (2007)

15. D.E. Gremyachkin, V.M. Piksaikin, K.V. Mitrofanov, A.S. Egorov, Measurements of temporal characteristics of delayed neutrons from neutron induced fission of ${ }^{237} \mathrm{~Np}$ in energy range from 14.2 to $18 \mathrm{MeV}$, EPJ Web Conf. 146, 04059 (2017)

16. V.A. Roshchenko, V.M. Piksaikin, L.E. Kazakov, G.G. Korolev, Relative yield of delayed neutrons and half-life of their precursor nuclei with fissioning of ${ }^{239} \mathrm{Pu}$ by $14.2-17.9$ MeV neutrons, At. Energy 101, 897 (2006)

17. D.E. Gremyachkin, V.M. Piksaikin, A.S. Egorov, K.V. Mitrofanov, Measurement of the temporal characteristics of delayed neutrons from neutron induced fission of ${ }^{241} \mathrm{Am}$ in the energy range from 14.2 to $18 \mathrm{MeV}$, to be published in Proceedings of the seminar ISINN-25, Dubna, Russian Federation 2017

18. J.N. Anoussis, D.C. Perricos, N.G. Chrysochoides, C.A. Mitsonias, Relative abundances for six delayed neutron groups from reactor neutron-induced fission of $231 \mathrm{~Pa}$, Radiochim. Acta 20, 118 (1973)

19. M.G. Brown, S.J. Lyle, E.B.M. Martin, Delayed neutron yields from $14.8 \mathrm{MeV}$ neutron induced fission of ${ }^{238} \mathrm{U},{ }^{232} \mathrm{Th}$ and ${ }^{231} \mathrm{~Pa}$, Radiochim. Acta 15, 109 (1971)

20. L.V. East, R.H. Augustson, H.O. Menlove, Delayed neutron abundances and half-lives from $14.7 \mathrm{MeV}$ fission nuclear safeguards research and development LA-4605-MS (1971)

21. G. Benedetti, A. Cesana, V. Sangiust, M. Terrani, Delayed neutron yields from fission of uranium-233, neptunium-237, plutonium-238, -240, -241, and americium-241, Nucl. Sci. Eng. 80, 379 (1982)

22. S.A. Cox, Delayed-neutron studies from the thermalneutron-induced fission of $241 \mathrm{Pu}$, Phys. Rev. 123 (1961)

23. A.N. Gudkov et al., Measurement of the delayed-neutron yields in the fission of ${ }^{233} \mathrm{U},{ }^{236} \mathrm{U},{ }^{237} \mathrm{~Np},{ }^{240} \mathrm{Pu}$ and ${ }^{241} \mathrm{Pu}$ by neutrons from the spectrum of a fast reactor. At. Energy. 66, $100(1989)$

24. W.S. Charlton, T.A. Parish, S. Raman, N. Shinohara, M. Andoh, Delayed Neutron Emission Measurements From Fast Fission Of U-235 and NP-237, CONF-960924-6 (1997)

25. E.T. Chulick, P.L. Reeder, Redetermination of delayed neutrons from ${ }^{252} \mathrm{Cf}$, Radiochimica Acta 12 (1969)

26. IAEA Reference Database for Beta-Delayed Neutron Emission https://www-nds.iaea.org/beta-delayed-neutron/ database.html

Cite this article as: Vladimir M. Piksaikin, Andrey S. Egorov, Dmitrii E. Gremyachkin, Konstantin V. Mitrofanov, Vjacheslav F. Mitrofanov, Generation of correlation and covariance matrices for the recommended temporal DN parameters in 6- and 8-group models, EPJ Nuclear Sci. Technol. 6, 54 (2020) 


\section{Appendix A Detailed description of the process of constants determination}

First of all we would like to note that we fix the relative abundances of the 1-st group to 1 . And then we make estimation of the relative abundances of the $2,3,4,5,6$ groups as a ratio to the value of the 1-st group $(=1)$ (see description of algorithm).

In order to clarify the estimation procedure we would propose consider the processing of experimental data obtained in one of experimental run for thermal induced fission of U235 . There were 12 cycles of irradiation. Time of transportation of sample $0.1 \mathrm{~s}$. Irradiation time $-180 \mathrm{~s}$. Counting time $700 \mathrm{~s}$. The decay curve is presented in Figure 1.

Processing procedure can be expressed in the following steps:

- A priori set of delayed neutron parameters can be taken from any available set from the literature (program is working even with all parameters which are equal to 1 ).

- Least square fit (LSF) of the decay curve is made in the range 10-700 s in order to estimate a1, a2, da2, T1, dT1, $\mathrm{T} 2$, dT2. $(\mathrm{da} 1=0$ means that a 1 is not free parameter $=$ 1). In LSF fitting parameters A (activity) and B (background) estimated as well.

- LSF fit of the decay curve in the range $0.1-700 \mathrm{~s}$ is made in order to estimate value of a3-6, da3-6 and T3-6, dT3-6 with fixed values of a1, a2, T1, T2 obtained in previous step.

As a result we have obtained the following set of DN parameters (ser $\# 1)$.

Transferring this data in commonly used form one have DN data set $\left(\Sigma a_{i}=1\right)$.

4) Using this set of DN parameters as a priori set we made another LSF fit of decay curve in the range

Table A1. Temporal DN parameters obtained in the result of the steps 1-3 (group yields are obtained relatively to the yield of the first group).

\begin{tabular}{|c|c|c|c|c|}
\hline & $\begin{array}{l}\text { A } \\
\text { Activity } \\
5547.61900\end{array}$ & 0.00574 & $\begin{array}{l}B \\
\text { Background } \\
0.23156\end{array}$ & 0.04951 \\
\hline$j$ & $t$ & $d t$ (relative) & $y$ & $d y$ (relative) \\
\hline 1 & 55.65603 & .00637 & 1.00000 & .00000 \\
\hline 2 & 22.41469 & .00781 & 6.48469 & .02200 \\
\hline 3 & 6.27364 & .03066 & 5.75592 & .04226 \\
\hline 4 & 2.27844 & .02123 & 11.44159 & .03596 \\
\hline 5 & 0.60671 & .04827 & 3.44605 & .04727 \\
\hline 6 & 0.22953 & .04985 & 1.26825 & .04971 \\
\hline
\end{tabular}

$\left(\mathrm{dT} 1 \mathrm{abs}=55.65603^{*} 0.00637=0.3546, \mathrm{dT} 2\right.$ abs $\ldots$ etc $)$.
$0.1-700$ s to get correlation matrix \#1 (all parameters are free but $\mathrm{a} 1=1$, da $1=0$ ).

It is seen that correlation coefficients cor(a1,T1), $\operatorname{cor}(\mathrm{a} 1, \mathrm{a} 2), \ldots=0$. Zero value of these correlation coefficients is not "set" to " 0 ". This is natural result of the LSF algorithm when we set zero value to uncertainty da1 in the a priori DN set (that means also that a1 is fixed).

Namely this type data set\#1 and Matrix $\# 1$ is used as a final set of data after estimation of uncertainty da1.

Estimation of the uncertainties da1 was made in the following procedure:

5) In order to estimate uncertainty of the relative abundance of 1-st group we made LSF fit of the decay curve in the range 0.1-700 s (as in step 4) with free all parameters including a1 (da1 is not equal to 0$)$. As a result of this step we have obtained the following DN parameters (set\#2) and appropriate correlation matrix \#2.

Transferring this data in commonly used form one have

Taking into account that sets $\# 1$ and $\# 2$ of DN parameters (including uncertainties) are very close one can say that estimation of da1 is reliable.

Moreover comparison of covariance matrix \#1 (with da1 $=0$, a1 is fixed to 1 ) with covariance matrix \#2 (with a1 as a free parameter) shows very close values of correlation coefficients. This is direct indication that procedure which we used for calculation of correlation matrix is correct. Using the procedure for calculating DN parameters with respect to the first group does not distort the correlation coefficient. There is no "impact on determination of the other parameters".

At that we adduce the correlation matrix obtained namely by calculation of the parameters relatively to fixed first group, since that in this case the algorithm gives the most correct solution.

Table A2. Temporal DN parameters in more common form (with absolute $d T$ and normalized $a_{i}$ values).

\begin{tabular}{|c|c|c|c|c|}
\hline$j$ & $\mathrm{~T}$ & $\mathrm{dT}$ & $\mathrm{y}$ & dy \\
\hline 1 & 55.65603 & .35460 & .03402 & .000 \\
\hline 2 & 22.41469 & .17501 & .22059 & .00485 \\
\hline 3 & 6.27364 & .19233 & .19580 & .00827 \\
\hline 4 & 2.27844 & .04838 & .38922 & .01399 \\
\hline 5 & 0.60671 & .02929 & .11723 & .00554 \\
\hline 6 & 0.22953 & .01144 & .04314 & .00214 \\
\hline \multicolumn{2}{|c|}{ Average half-life } & 9.034057 & & \\
\hline
\end{tabular}


Table A3. Correlation matrix obtained in the result of the steps 1-4 (a1 is fixed).

\begin{tabular}{|c|c|c|c|c|c|c|c|c|c|c|c|c|c|c|}
\hline & A & B & a1 & $\mathrm{T} 1$ & $\mathrm{a} 2$ & $\mathrm{~T} 2$ & a3 & T3 & a4 & $\mathrm{T} 4$ & a5 & $\mathrm{T} 5$ & $\mathrm{a} 6$ & $\mathrm{~T} 6$ \\
\hline A & 1 & & & & & & & & & & & & & \\
\hline B & 0.02 & 1 & & & & & & & & & & & & \\
\hline a1 & 0 & 0 & 1 & & & & & & & & & & & \\
\hline $\mathrm{T} 1$ & -0.31 & -0.07 & 0 & 1 & & & & & & & & & & \\
\hline $\mathrm{a} 2$ & -0.31 & -0.05 & 0 & 0.86 & 1 & & & & & & & & & \\
\hline $\mathrm{T} 2$ & -0.24 & -0.01 & 0 & 0.51 & 0.31 & 1 & & & & & & & & \\
\hline a3 & -0.04 & -0.02 & 0 & 0.38 & 0.4 & 0.34 & 1 & & & & & & & \\
\hline T3 & -0.12 & 0 & 0 & 0.23 & -0.07 & 0.68 & -0.3 & 1 & & & & & & \\
\hline a4 & -0.16 & -0.03 & 0 & 0.68 & 0.57 & 0.7 & -0.04 & 0.68 & 1 & & & & & \\
\hline $\mathrm{T} 4$ & -0.35 & 0 & 0 & 0.06 & 0.06 & 0.04 & -0.63 & 0.25 & 0.17 & 1 & & & & \\
\hline a5 & 0.33 & -0.01 & 0 & 0.14 & 0.14 & 0.11 & -0.02 & 0.06 & -0.04 & 0.32 & 1 & & & \\
\hline T5 & -0.42 & 0 & 0 & 0.04 & 0.03 & 0.04 & 0 & 0.04 & -0.12 & 0.14 & 0.03 & 1 & & \\
\hline $\mathrm{a} 6$ & 0.25 & 0 & 0 & 0.03 & 0.03 & 0.02 & 0 & 0.01 & 0.01 & 0.05 & -0.03 & 0.02 & 1 & \\
\hline T6 & -0.12 & 0 & 0 & 0.02 & 0.03 & 0.02 & 0 & 0.01 & 0 & 0.06 & -0.02 & 0.01 & -0.01 & 1 \\
\hline
\end{tabular}

Table A4. Temporal DN parameters obtained in the result of the steps 1-3 using set\#1 as a priori data (group yields are obtained relatively to the yield of the first group).

\begin{tabular}{lllll}
\hline & $\begin{array}{l}A \\
\text { activity }\end{array}$ & $d A$ & $\begin{array}{l}B \\
\text { background } \\
0.18002\end{array}$ & $d B$ \\
\hline$j$ & 5551.80400 & 0.00582 & $y$ & 0.04971 \\
1 & $t$ & $d t$ (relative) & 1.00242 & .00978 \\
2 & 55.43494 & .00648 & 6.41151 & .02195 \\
3 & 22.27870 & .00822 & 5.63918 & .04190 \\
4 & 6.22071 & .03113 & 11.22377 & .03584 \\
5 & 2.28102 & .02169 & 3.41686 & .04721 \\
6 & 0.60578 & .04820 & 1.26605 & .04970 \\
\hline
\end{tabular}

Table A5. Temporal DN parameters in more common form (with absolute $d T$ and normalized $a_{i}$ values).

\begin{tabular}{lllll}
\hline$j$ & $T$ & $d T$ & $y$ & $d y$ \\
\hline 1 & 55.43494 & .35909 & .03461 & .00076 \\
2 & 22.27870 & .18316 & .22139 & .00486 \\
3 & 6.22071 & .19366 & .19472 & .00816 \\
4 & 2.28102 & .04948 & .38756 & .01389 \\
5 & 0.60578 & .02920 & .11799 & .00557 \\
6 & 0.22856 & .01139 & .04372 & .00217 \\
Average half-life & & 9.028021 & & \\
\hline
\end{tabular}


Table A6. Correlation matrix obtained using all free parameters.

\begin{tabular}{|c|c|c|c|c|c|c|c|c|c|c|c|c|c|c|}
\hline & A & B & a1 & $\mathrm{T} 1$ & $\mathrm{a} 2$ & $\mathrm{~T} 2$ & a3 & $\mathrm{T} 3$ & $\mathrm{a} 4$ & $\mathrm{~T} 4$ & a5 & $\mathrm{T} 5$ & a6 & $\mathrm{T} 6$ \\
\hline A & 1 & & & & & & & & & & & & & \\
\hline B & 0.01 & 1 & & & & & & & & & & & & \\
\hline a1 & -0.06 & 0.01 & 1 & & & & & & & & & & & \\
\hline $\mathrm{T} 1$ & -0.28 & -0.06 & -0.25 & 1 & & & & & & & & & & \\
\hline $\mathrm{a} 2$ & -0.31 & -0.04 & 0.19 & 0.76 & 1 & & & & & & & & & \\
\hline $\mathrm{T} 2$ & -0.23 & -0.01 & -0.2 & 0.55 & 0.23 & 1 & & & & & & & & \\
\hline a3 & -0.05 & -0.01 & 0.04 & 0.37 & 0.39 & 0.34 & 1 & & & & & & & \\
\hline T3 & -0.12 & 0 & -0.05 & 0.24 & -0.11 & 0.68 & -0.29 & 1 & & & & & & \\
\hline a4 & -0.16 & -0.02 & 0.1 & 0.63 & 0.54 & 0.66 & -0.03 & 0.67 & 1 & & & & & \\
\hline $\mathrm{T} 4$ & -0.34 & 0 & 0 & 0.06 & 0.06 & 0.05 & -0.62 & 0.27 & 0.17 & 1 & & & & \\
\hline a5 & 0.33 & -0.01 & 0.03 & 0.13 & 0.14 & 0.11 & -0.01 & 0.06 & -0.04 & 0.33 & 1 & & & \\
\hline T5 & -0.42 & 0 & 0.01 & 0.04 & 0.03 & 0.04 & 0 & 0.04 & -0.13 & 0.13 & 0.03 & 1 & & \\
\hline $\mathrm{a} 6$ & 0.25 & 0 & 0.01 & 0.03 & 0.03 & 0.02 & 0 & 0.01 & 0.01 & 0.05 & -0.03 & 0.02 & 1 & \\
\hline T6 & -0.12 & 0 & 0 & 0.02 & 0.03 & 0.02 & 0 & 0.01 & 0 & 0.06 & -0.02 & 0.01 & -0.01 & 1 \\
\hline
\end{tabular}

\section{Appendix B Motivation for procedure used in our approach}

In order to obtain the 8 group DN parameters values and uncertainties comparable with 6 -group data we need to get very good statistics (it is because of 8 group parameters in 8 group model as compared with 12 group parameters of 6 group model). It is quite expensive to make experiments on the electrostatic accelerator. Besides to get reliable data we should make many cycles of measurements with stable ion current and stable neutron target (it can be deteriorated by ion flux).

From our experience some existing "raw data" gave biased estimation in $\langle T\rangle$ relatively to the 6 -group model. In addition there is a need to develop averaging procedure for 8-group data that we have for 6-group data. Therefore we decided to follow approach used in Spriggs et al paper [3]: to calculate "model decay curve" on the basis of 6 group data. (In the present work we used the data obtained after averaging procedures. So there was no need to have 8-group averaging procedure) and process it by the LSF. Many tests were made. It turns out that in this approach we have better estimation of $\langle T\rangle$ as compared with "raw data" processing with comparable uncertainties $\mathrm{d}<T>$.

\section{Appendix C Variation of the input conditions (activity, background)}

Using the data from Table A1 we made for the example datasets with $A=1000, B=100$ and $A=5000, B=100$ to show the influence of less favorable background conditions.

It has the following correlation matrix:

Using «input dataset» the decay curve has been obtained. It has been used to obtain dataset and correlation matrix (as an example of obtaining correlation matrices from another works).

And appropriate correlation matrix, which is almost identical to the «input» one.

Also using the data from we have we made for the example dataset with $(\mathrm{A}=5000, \mathrm{~B}=100)$.

It has the following correlation matrix.

Using «input dataset» the decay curve has been obtained. It has been used to obtain dataset and correlation matrix (as an example of obtaining correlation matrices from another works). 
Table C1. "Input dataset" for testing the quality of generation of correlation matrices $(A=1000, B=100)$.

\begin{tabular}{lllll}
\hline & $A$ & $d A$ & $B$ & $d B$ \\
\hline$j$ & 1004 & 21.814 & 99.978 & 0.474 \\
1 & $t$ & $d_{t}$ & $a_{i}$ & $d a_{i}$ \\
2 & 53.78 & 2.554 & 0.038 & 0.002 \\
3 & 22.28 & 0.6814 & 0.2126 & 0.01 \\
4 & 6.426 & 0.3002 & 0.1971 & 0.01 \\
5 & 2.268 & 0.1052 & 0.3947 & 0.018 \\
6 & 0.495 & 0.0247 & 0.1316 & 0.007 \\
Average half-life & 0.179 & 0.009 & 0.026 & 0.001 \\
\hline
\end{tabular}

Table C2. Correlation matrix appropriate to the input dataset $\left(a_{i}, T_{i}\right)(A=1000, B=100)$.

\begin{tabular}{|c|c|c|c|c|c|c|c|c|c|c|c|c|c|c|}
\hline & $\mathrm{A}$ & B & a1 & $\mathrm{T} 1$ & $\mathrm{a} 2$ & $\mathrm{~T} 2$ & a3 & T3 & $\mathrm{a} 4$ & $\mathrm{~T} 4$ & a5 & $\mathrm{T} 5$ & $\mathrm{a} 6$ & $\mathrm{~T} 6$ \\
\hline A & 1 & & & & & & & & & & & & & \\
\hline B & -0.08 & 1 & & & & & & & & & & & & \\
\hline a1 & 0 & 0 & 1 & & & & & & & & & & & \\
\hline $\mathrm{T} 1$ & 0.02 & -0.27 & 0 & 1 & & & & & & & & & & \\
\hline $\mathrm{a} 2$ & -0.37 & 0.05 & 0 & 0.05 & 1 & & & & & & & & & \\
\hline $\mathrm{T} 2$ & -0.22 & -0.18 & 0 & -0.17 & -0.25 & 1 & & & & & & & & \\
\hline a3 & -0.09 & 0.02 & 0 & 0.01 & 0.04 & 0.22 & 1 & & & & & & & \\
\hline T3 & -0.21 & 0.02 & 0 & 0.03 & -0.15 & 0.08 & -0.01 & 1 & & & & & & \\
\hline $\mathrm{a} 4$ & 0.24 & 0.03 & 0 & 0 & 0.2 & 0.23 & -0.02 & 0.13 & 1 & & & & & \\
\hline $\mathrm{T} 4$ & -0.39 & 0.01 & 0 & 0 & 0.03 & 0.19 & -0.07 & -0.01 & -0.01 & 1 & & & & \\
\hline a 5 & 0.22 & 0 & 0 & 0 & 0.03 & 0.02 & 0 & 0.02 & -0.03 & 0.03 & 1 & & & \\
\hline T5 & -0.06 & 0 & 0 & 0 & 0.02 & 0.02 & 0 & 0.01 & -0.02 & 0.01 & 0 & 1 & & \\
\hline $\mathrm{a} 6$ & 0.05 & 0 & 0 & 0 & 0 & 0 & 0 & 0 & 0 & 0 & 0 & 0 & 1 & \\
\hline T6 & -0.01 & 0 & 0 & 0 & 0 & 0 & 0 & 0 & 0 & 0 & 0 & 0 & 0 & 1 \\
\hline
\end{tabular}

Table C3. The 6-group DN parameters $\left(a_{i}, T_{i}\right)$ obtained in the LSF processing of the model decay curve calculated with the help of the 6-group DN "input dataset" $(A=1000 ; B=100)$.

\begin{tabular}{lllll}
\hline & $\mathrm{A}$ & $\mathrm{dA}$ & $\mathrm{B}$ & $\mathrm{dB}$ \\
\hline$j$ & 999.2 & 21.735 & 99.9922 & 0.474 \\
1 & $t$ & $d t$ & $y$ & $d y$ \\
2 & 53.97 & 2.5643 & 0.03803 & 0.002 \\
3 & 22.31 & 0.6834 & 0.21249 & 0.009 \\
4 & 6.431 & 0.3005 & 0.19748 & 0.01 \\
5 & 2.273 & 0.1055 & 0.39436 & 0.018 \\
6 & 0.494 & 0.0247 & 0.13165 & 0.007 \\
Average half-life & 0.179 & 0.009 & 0.026 & 0.001 \\
\hline
\end{tabular}


Table C4. The 6-group correlation matrix obtained in processing of the model decay curve calculated with the help of the 6-group DN "input dataset" $(A=1000 ; B=100)$.

\begin{tabular}{|c|c|c|c|c|c|c|c|c|c|c|c|c|c|c|}
\hline & A & B & a1 & $\mathrm{T} 1$ & $\mathrm{a} 2$ & $\mathrm{~T} 2$ & a3 & T3 & $\mathrm{a} 4$ & $\mathrm{~T} 4$ & a5 & $\mathrm{T} 5$ & $\mathrm{a} 6$ & $\mathrm{~T} 6$ \\
\hline A & 1 & & & & & & & & & & & & & \\
\hline B & -0.08 & 1 & & & & & & & & & & & & \\
\hline a1 & 0 & 0 & 1 & & & & & & & & & & & \\
\hline $\mathrm{T} 1$ & 0.02 & -0.27 & 0 & 1 & & & & & & & & & & \\
\hline $\mathrm{a} 2$ & -0.37 & 0.05 & 0 & 0.05 & 1 & & & & & & & & & \\
\hline $\mathrm{T} 2$ & -0.22 & -0.18 & 0 & -0.17 & -0.25 & 1 & & & & & & & & \\
\hline a3 & -0.09 & 0.02 & 0 & 0.01 & 0.04 & 0.22 & 1 & & & & & & & \\
\hline T3 & -0.21 & 0.02 & 0 & 0.03 & -0.15 & 0.08 & -0.01 & 1 & & & & & & \\
\hline $\mathrm{a} 4$ & 0.24 & 0.03 & 0 & 0 & 0.2 & 0.23 & -0.03 & 0.13 & 1 & & & & & \\
\hline $\mathrm{T} 4$ & -0.39 & 0.01 & 0 & 0 & 0.03 & 0.19 & -0.07 & -0.01 & -0.01 & 1 & & & & \\
\hline a5 & 0.22 & 0 & 0 & 0 & 0.03 & 0.02 & 0 & 0.02 & -0.03 & 0.03 & 1 & & & \\
\hline $\mathrm{T} 5$ & -0.06 & 0 & 0 & 0 & 0.02 & 0.02 & 0 & 0.01 & -0.02 & 0.01 & 0 & 1 & & \\
\hline $\mathrm{a} 6$ & 0.05 & 0 & 0 & 0 & 0 & 0 & 0 & 0 & 0 & 0 & 0 & 0 & 1 & \\
\hline T6 & -0.01 & 0 & 0 & 0 & 0 & 0 & 0 & 0 & 0 & 0 & 0 & 0 & 0 & 1 \\
\hline
\end{tabular}

Table C5. "Input dataset" for testing the quality of generation of correlation matrices $(A=5000, B=100)$.

\begin{tabular}{lllll}
\hline & $\mathrm{A}$ & $\mathrm{dA}$ & $\mathrm{B}$ & $\mathrm{dB}$ \\
\hline$j$ & 5027 & 65.684 & 100.0935 & 0.582 \\
1 & $t$ & $d t$ & $y$ & $d y$ \\
2 & 53.07 & 1.7068 & 0.0379 & 0.001 \\
3 & 22.19 & 0.4173 & 0.21391 & 0.008 \\
4 & 6.386 & 0.2686 & 0.19544 & 0.009 \\
5 & 2.252 & 0.0847 & 0.39561 & 0.017 \\
6 & 0.495 & 0.0246 & 0.13124 & 0.006 \\
Average half-life & 0.179 & 0.009 & 0.02591 & 0.001 \\
\hline
\end{tabular}

Table C6. Correlation matrix appropriate to the input dataset $\left(a_{i}, T_{i}\right)(A=5000, B=100)$.

\begin{tabular}{|c|c|c|c|c|c|c|c|c|c|c|c|c|c|c|}
\hline & $\mathrm{A}$ & B & a1 & $\mathrm{T} 1$ & $\mathrm{a} 2$ & $\mathrm{~T} 2$ & a3 & T3 & $\mathrm{a} 4$ & $\mathrm{~T} 4$ & $\mathrm{a} 5$ & T5 & $\mathrm{a} 6$ & $\mathrm{~T} 6$ \\
\hline $\mathrm{A}$ & 1 & & & & & & & & & & & & & \\
\hline B & -0.05 & 1 & & & & & & & & & & & & \\
\hline a1 & 0 & 0 & 1 & & & & & & & & & & & \\
\hline $\mathrm{T} 1$ & 0.05 & -0.55 & 0 & 1 & & & & & & & & & & \\
\hline $\mathrm{a} 2$ & -0.31 & -0.01 & 0 & 0.33 & 1 & & & & & & & & & \\
\hline $\mathrm{T} 2$ & -0.22 & 0.12 & 0 & -0.43 & -0.3 & 1 & & & & & & & & \\
\hline a3 & -0.12 & 0.03 & 0 & 0 & 0.09 & 0.39 & 1 & & & & & & & \\
\hline T3 & -0.12 & 0 & 0 & 0 & -0.33 & 0.4 & -0.04 & 1 & & & & & & \\
\hline a4 & 0.07 & 0.04 & 0 & 0.09 & 0.4 & 0.32 & 0 & 0.34 & 1 & & & & & \\
\hline $\mathrm{T} 4$ & -0.53 & 0.03 & 0 & -0.03 & 0.08 & 0.25 & -0.22 & 0.03 & 0.04 & 1 & & & & \\
\hline $\mathrm{a} 5$ & 0.28 & 0.01 & 0 & 0.01 & 0.06 & 0.04 & 0.02 & 0.04 & -0.05 & 0.1 & 1 & & & \\
\hline T5 & -0.16 & 0 & 0 & 0.01 & 0.04 & 0.02 & 0.01 & 0.03 & -0.05 & 0.04 & 0 & 1 & & \\
\hline $\mathrm{a} 6$ & 0.08 & 0 & 0 & 0 & 0.01 & 0 & 0 & 0 & 0 & 0.01 & 0 & 0 & 1 & \\
\hline $\mathrm{T} 6$ & -0.02 & 0 & 0 & 0 & 0 & 0 & 0 & 0 & 0 & 0.01 & 0 & 0 & 0 & 1 \\
\hline
\end{tabular}


Table C7. The 6-group DN parameters $\left(a_{i}, T_{i}\right)$ obtained in the LSF processing of the model decay curve calculated with the help of the 6 -group DN "input dataset" $(A=5000 ; B=100)$.

\begin{tabular}{lllll}
\hline & $A$ & $d A$ & $B$ & $d B$ \\
\hline$j$ & 4998.8 & 65.43668 & 99.97 & 0.582 \\
1 & $t$ & $d t$ & $y$ & $d y$ \\
2 & 53.169 & 1.71257 & 0.038 & 0.001 \\
3 & 22.217 & 0.41897 & 0.213 & 0.008 \\
4 & 6.4165 & 0.26969 & 0.196 & 0.009 \\
5 & 2.2534 & 0.08488 & 0.395 & 0.017 \\
6 & 0.4947 & 0.02455 & 0.132 & 0.006 \\
Average half-life & 0.179 & 0.00895 & 0.026 & 0.001 \\
\hline
\end{tabular}

Table C8. The 6-group correlation matrix obtained in processing of the model decay curve calculated with the help of the 6-group DN "input dataset" $(A=5000 ; B=100)$.

\begin{tabular}{|c|c|c|c|c|c|c|c|c|c|c|c|c|c|c|}
\hline & $\mathrm{A}$ & B & a1 & $\mathrm{T} 1$ & $\mathrm{a} 2$ & $\mathrm{~T} 2$ & a3 & $\mathrm{T} 3$ & $\mathrm{a} 4$ & $\mathrm{~T} 4$ & $\mathrm{a} 5$ & T5 & $\mathrm{a} 6$ & $\mathrm{~T} 6$ \\
\hline $\mathrm{A}$ & 1 & & & & & & & & & & & & & \\
\hline B & -0.05 & 1 & & & & & & & & & & & & \\
\hline a1 & 0 & 0 & 1 & & & & & & & & & & & \\
\hline $\mathrm{T} 1$ & -0.05 & -0.55 & 0 & 1 & & & & & & & & & & \\
\hline $\mathrm{a} 2$ & -0.31 & -0.01 & 0 & 0.33 & 1 & & & & & & & & & \\
\hline $\mathrm{T} 2$ & -0.22 & 0.12 & 0 & -0.42 & -0.3 & 1 & & & & & & & & \\
\hline a3 & -0.12 & 0.03 & 0 & 0 & 0.09 & 0.39 & 1 & & & & & & & \\
\hline T3 & -0.12 & 0 & 0 & 0 & -0.33 & 0.4 & -0.04 & 1 & & & & & & \\
\hline $\mathrm{a} 4$ & 0.07 & 0.04 & 0 & 0.09 & 0.4 & 0.32 & 0 & 0.34 & 1 & & & & & \\
\hline $\mathrm{T} 4$ & -0.53 & 0.03 & 0 & -0.03 & 0.08 & 0.25 & -0.21 & 0.03 & 0.04 & 1 & & & & \\
\hline a 5 & 0.28 & 0.01 & 0 & 0.01 & 0.06 & 0.04 & 0.02 & 0.04 & -0.05 & 0.1 & 1 & & & \\
\hline T5 & -0.16 & 0 & 0 & 0.01 & 0.04 & 0.02 & 0.01 & 0.03 & -0.05 & 0.04 & 0 & 1 & & \\
\hline a6 & 0.08 & 0 & 0 & 0 & 0.01 & 0 & 0 & 0 & 0 & 0.01 & 0 & 0 & 1 & \\
\hline T6 & -0.02 & 0 & 0 & 0 & 0 & 0 & 0 & 0 & 0 & 0.01 & 0 & 0 & 0 & 1 \\
\hline
\end{tabular}

